\title{
Effect of rotation on ferromagnetic porous convection with a thermal non-equilibrium model
}

\author{
I.S. Shivakumara $\cdot$ R. Gangadhara Reddy • \\ M. Ravisha $\cdot$ Jinho Lee
}

Received: 18 November 2012 / Accepted: 9 December 2013 / Published online: 18 March 2014

(C) Springer Science+Business Media Dordrecht 2014

\begin{abstract}
The effect of rotation on the onset of thermal convection in a horizontal layer of ferrofluid saturated Brinkman porous medium is investigated in the presence of a uniform vertical magnetic field using a local thermal non-equilibrium (LTNE) model. A twofield model for temperature representing the solid and fluid phases separately is used for energy equation. The condition for the occurrence of stationary and oscillatory convection is obtained analytically. The stability of the system has been analyzed when the magnetic and buoyancy forces are acting together as well as in isolation and the similarities as well as differ-
\end{abstract}

\footnotetext{
I.S. Shivakumara $(\varangle)$

UGC-Centre for Advanced Studies in Fluid Mechanics, Department of Mathematics, Bangalore University,

Bangalore 560 001, India

e-mail: shivakumarais@gmail.com

R. Gangadhara Reddy

Department of Mathematics, AMC Engineering College,

Bangalore 560 083, India

e-mail: greddypulakurthi@gmail.com

\section{Ravisha}

Department of Mathematics, Dr. G. Shankar Government Women's First Grade College and Post Graduate Study Centre, Udupi 576101, India

e-mail: ravishmamatha@gmail.com

J. Lee

School of Mechanical Engineering, Yonsei University, Seoul 120-749, South Korea

e-mail: jinholee@yonsei.ac.kr
}

ences between the two are highlighted. In contrast to the non-rotating case, it is shown that decrease in the Darcy number $D a$ and an increase in the ratio of effective viscosity to fluid viscosity $\Lambda$ is to hasten the onset of stationary convection at high rotation rates and a coupling between these two parameters is identified in destabilizing the system. Asymptotic solutions for both small and large values of scaled interphase heat transfer coefficient $H_{t}$ are presented and compared with those computed numerically. Besides, the influence of magnetic parameters and also parameters representing LTNE on the stability of the system is discussed and the veracity of LTNE model over the LTE model is also analyzed.

Keywords Ferromagnetic convection · Porous medium $\cdot$ Rotation $\cdot$ Thermal non-equilibrium model

\section{Introduction}

Ferromagnetic fluids are colloidal suspensions of permanently magnetized nanoparticles in carrier liquids like water, heptane, kerosene or various oils. The nanoparticles typically have sizes of about $3 \sim 10 \mathrm{~nm}$ and each particle is encapsulated by a surfactant to provide short range steric repulsion between particles to prevent particle agglomeration in the presence of magnetic field. These fluids are also termed as magnetic fluids or magnetic nanofluids and they are not found in nature but are artificially synthesized in the 
laboratory. The peculiarity of ferrofluids is the combination of normal liquid behavior with a magnetic control of their flow and properties. This possibility originates from the fact that each of the particles can be treated as a thermally agitated single domain particle in the carrier liquid. The continuum description of the ferrofluid flow termed as ferrohydrodynamics has been in existence since 1960s and the field of ferrofluid research has gained its momentum over the years because of the importance of these wonder fluids in many technological applications of commercial value (Rosensweig [1]; Berkovsky et al. [2]; Blums et al. [3]; Hergt et al. [4]; Alexiou et al. [5]).

Thermal convection of ferrofluids in non-porous and porous domains heated uniformly from below in the presence of a uniform magnetic field, known as ferromagnetic convection, has attracted considerable attention in the literature. Ganguly et al. [6] have given an overview of prior research on heat transfer in ferrofluid flows and also discussed the heat transfer augmentation due to the thermomagnetic convection. In his review article, Odenbach [7] has focused on recent developments in the field of rheological investigations of ferrofluids and their importance for the general treatment of ferrofluids. Thermal convective instability in a ferrofluid saturated porous domain has also been investigated extensively owing to their importance in controlled emplacement of liquids or treatment of chemicals, and emplacement of geophysically imageable liquids into particular zones for subsequent imaging etc. Rosensweig et al. [8] have studied experimentally the penetration of ferrofluids in the HeleShaw cell. The stability of the magnetic fluid penetration through a porous medium in high uniform magnetic field oblique to the interface is studied by Zahn and Rosensweig [9]. The thermal convection of ferrofluid saturating a porous medium in the presence of a vertical magnetic field is studied by Vaidyanathan et al. [10] by employing the Brinkman equation. The experimental results of the behavior of ferrofluids in porous media consisting of sands and sediments are presented in detail by Borglin et al. [11]. Sunil and Mahajan [12] have used generalized energy method to study nonlinear convection in a magnetized ferrofluid saturated porous layer heated uniformly from below for the stress-free boundaries case. Shivakumara et al. $[13,14]$ have investigated theoretically the onset of convection in a layer of ferrofluid saturated porous medium for various types of velocity and temperature boundary conditions. Nanjundappa et al. [15] have investigated the onset of buoyancy-driven convection in a ferromagnetic fluid saturated sparsely packed porous medium with fixed heat flux condition at the lower rigid boundary and a general thermal boundary condition at the upper free boundary.

In many practical applications involving hyperporous materials and also media in which there is a large temperature difference between the fluid and solid phases, it has been realized that the assumption of local thermal equilibrium (LTE) is inadequate for proper understanding of the heat transfer problems. In such circumstances, the local thermal nonequilibrium (LTNE) effects are to be taken into consideration. Therefore, the recent trend in the study of thermal convective instability problems in porous media is to account for LTNE effects by considering a two-field model for energy equation each representing the fluid and solid phases separately. Copious literature is available on natural convection in an ordinary viscous fluid saturating a porous layer using a LTNE model (Rees and Pop [16], Nield and Bejan [17]; Shivakumara et al. [18] and references therein). Nonetheless, limited attention has been given to assess LTNE effects on the criterion for the onset of ferromagnetic convection in a porous medium despite its relevance and importance in many heat transfer problems as mentioned above [19-22].

Ferrofluids are known to exhibit strange characteristics when they are set to rotation and hence investigating the effects of rotation on ferromagnetic convection in a rotating porous medium is scientifically and technologically important. Sekar et al. [23] and Vaidyanathan et al. [24] have discussed the effect of rotation on convective instability in a ferrofluid saturated porous layer heating uniformly from below. In the latter paper, the effect of magnetic field dependent viscosity is also taken into consideration. Subsequently, many researchers have extended these works to include various additional effects. Sunil and Mahajan [25] have performed nonlinear stability analysis for rotating magnetized ferrofluid heated from below saturating a porous medium for free boundaries. Shivakumara et al. [26] have studied the effect of Coriolis force on the onset of ferromagnetic convection in a rotating horizontal ferrofluid saturated porous layer in the presence of a uniform vertical magnetic field considering the bounding surfaces of the porous layer to be either stress-free or rigid. Contrary to their stabilizing effect in the absence of rotation, they have shown 
that increasing the ratio of viscosities $\Lambda$ and decreasing the Darcy number $D a$ hasten the onset of stationary ferromagnetic convection in the presence of rotation. All the above studies are based on LTE model.

The combined effect of rotation and LTNE on linear and nonlinear thermal convection in an ordinary viscous fluid saturating a porous medium has been studied by Straughan [27]. He has established the equivalence of the linear instability and nonlinear stability boundaries for the thermal convection in a rotating porous layer with the Darcy law using a LTNE model. Malashetty et al. [28] have studied linear and weakly nonlinear thermal convection in a rotating densely packed porous layer using a LTNE model. Nonetheless, the problem involving the simultaneous effect of rotation and LTNE on ferromagnetic convection has not been given due attention despite its importance in many heat transfer related problems except the study undertaken by Sunil et al. [29].

The intent of the present study is, therefore, to perform the linear stability analysis of thermal convection in a rotating sparsely packed horizontal ferromagnetic fluid saturated Brinkman porous layer using a LTNE model and to elucidate that the dynamical system considered is capable of supporting some new results not observed in the absence of rotation and also in the previous investigation. The criterion for the onset of stationary and oscillatory convection is obtained analytically and some interesting observations on the stability characteristics of the system have been unfolded through numerical calculations. In addition, the veracity of LTNE model over the LTE model is also analyzed. Besides, the asymptotic analysis has been carried out for very small and large values of interphase heat transfer coefficient to calculate critical Rayleigh and wave numbers. Since the ferromagnetic fluids are considered to be good carriers of heat, a possible engineering application of the findings of the current study may be useful in understanding the cooling of electronic circuits found in rotating radars. Besides, the study helps in understanding control of ferroconvection by the Coriolis force due to rotation.

\section{Formulation of the problem}

We consider an initially quiescent incompressible constant viscosity ferromagnetic fluid saturated horizontal porous layer of characteristic thickness $d$ in the presence of a uniform applied magnetic field $H_{0}$ in the vertical direction as shown in Fig. 1. The lower surface is

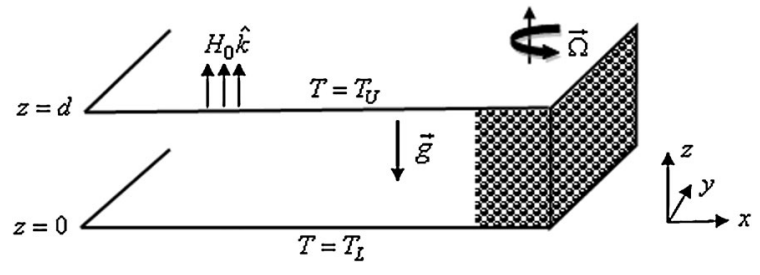

Fig. 1 Physical configuration

held at constant temperature $T_{L}$, while the upper surface is at $T_{U}\left(<T_{L}\right)$. A Cartesian co-ordinate system $(x, y, z)$ is used with the origin at the bottom of the porous layer and the $z$-axis directed vertically upward in the presence of gravitational field. The entire system is rotating with constant angular velocity $\vec{\Omega}=\Omega \hat{k}$, where $\hat{k}$ is the unit vector in the vertical direction. It is assumed that the rotation does not disrupt the isotropy of the porous medium. The flow in the porous medium is described by the Brinkman-extended-Darcy equation with fluid viscosity different from effective viscosity and the Boussinesq approximation on the density is made. The solid and fluid phases of the porous medium are assumed to be in LTNE and a two-field model for temperatures is used. The basic equations are:

$\nabla \cdot \vec{q}=0$

$$
\begin{aligned}
\rho_{0}\left[\frac{1}{\varepsilon}\right. & \left.\frac{\partial \vec{q}}{\partial t}+\frac{1}{\varepsilon^{2}}(\vec{q} \cdot \nabla) \vec{q}\right] \\
= & -\nabla\left(\frac{\rho_{0}}{2}(|\vec{\Omega} \times \vec{r}|)+p\right) \\
& +\rho_{f} \vec{g}-\frac{\mu_{f}}{k} \vec{q}+\tilde{\mu}_{f} \nabla^{2} \vec{q}+\mu_{0}(\vec{M} \cdot \nabla) \vec{H} \\
& +\frac{\rho_{0} c_{F}}{\sqrt{k}}|\vec{q}| \vec{q}+2 \frac{\rho_{0}}{\varepsilon}(\vec{q} \times \vec{\Omega})
\end{aligned}
$$

$$
\begin{aligned}
\varepsilon\left(\rho_{0} c\right)_{f} \frac{\partial T_{f}}{\partial t}+\left(\rho_{0} c\right)_{f}(\vec{q} \cdot \nabla) T_{f}= & \varepsilon k_{f} \nabla^{2} T_{f} \\
& +h\left(T_{s}-T_{f}\right)
\end{aligned}
$$

$$
\begin{aligned}
& (1-\varepsilon)\left(\rho_{0} c\right)_{s} \frac{\partial T_{s}}{\partial t}=(1-\varepsilon) k_{s} \nabla^{2} T_{s}-h\left(T_{s}-T_{f}\right) \\
& \rho_{f}=\rho_{0}\left[1-\alpha_{t}\left(T_{f}-T_{a}\right)\right]
\end{aligned}
$$

where, $\vec{q}$ the velocity vector, $p$ the pressure, $\vec{M}$ the magnetization, $\vec{H}$ the magnetic field intensity, $\rho_{f}$ the fluid density, $k$ the permeability of the porous medium, 
$c_{F}$ the dimensionless form drag constant, $\varepsilon$ the porosity of the porous medium, $\mu_{f}$ the fluid viscosity, $\tilde{\mu}_{f}$ is the effective viscosity, $\mu_{0}$ the magnetic permeability of vacuum, $T_{f}$ the temperature of the fluid phase, $T_{s}$ the temperature of the solid phase, $c$ the specific heat, $k_{f}$ the thermal conductivity of the fluid, $k_{s}$ the thermal conductivity of the solid, $h$ is the inter-phase heat transfer coefficient, $\rho_{0}$ the reference value, $\alpha_{t}$ the thermal expansion coefficient, $T_{a}=\left(T_{L}+T_{U}\right) / 2$ the average temperature. It may be noted that large values of $h$ correspond to a rapid transfer of heat between the phases which represents the LTE case, while moderate values of $h$ correspond to relatively strong LTNE effects. In other words, it measures the ease with which heat is transferred between the phases. In (3) and (4), $T_{f}$ and $T_{s}$ are intrinsic averages of the temperature fields, and this allows one to set $T_{f}=T_{s}=T_{b}$ whenever the boundary of the porous medium is maintained at the temperature $T_{b}$.

The Maxwell equations in the magnetostatic limit are:

$\nabla \cdot \vec{B}=0$

$\nabla \times \vec{H}=0 \quad$ or $\quad \vec{H}=\nabla \phi$

where, $\vec{B}$ is the magnetic induction and $\phi$ is the magnetic potential. Further, $\vec{B}, \vec{M}$ and $\vec{H}$ are related by

$\vec{B}=\mu_{0}(\vec{M}+\vec{H})$.

It is assumed that the magnetization is aligned with the magnetic field, but allowed a dependence on the magnitude of the magnetic field as well as temperature (Finlayson [30]) and thus

$\vec{M}=M\left(H, T_{f}\right) \frac{\vec{H}}{H}$

where $M=|\vec{M}|$ and $H=|\vec{H}|$. The magnetic equation of state, following (Finlayson [30]), is taken as

$M=M_{0}+\chi\left(H-H_{0}\right)-K\left(T_{f}-T_{a}\right)$

where, $\chi=(\partial M / \partial H)_{H_{0}, T_{a}}$, the magnetic susceptibility, $K=-\left(\partial M / \partial T_{f}\right)_{H_{0}, T_{a}}$, the pyromagnetic coefficient and $M_{0}=M\left(H_{0}, T_{a}\right)$.

\subsection{Basic state}

The basic state is quiescent and there exists the following solution for the basic state:

$\vec{q}_{b}=0$

$$
\begin{aligned}
p_{b}(z)= & p_{0}-\rho_{0} g z-\frac{1}{2} \rho_{0} \alpha_{t} g \beta z(z-d)-\frac{\mu_{0} M_{0} K \beta}{1+\chi} z \\
& -\frac{\mu_{0} K^{2} \beta^{2}}{2(1+\chi)^{2}} z(z-d) \\
T_{f b}(z)= & T_{a}-\beta(z-d / 2)=T_{s b}(z) \\
\vec{H}_{b}(z)= & {\left[H_{0}-\frac{K \beta}{1+\chi}\left(z-\frac{d}{2}\right)\right] \hat{k} } \\
\vec{M}_{b}(z)= & {\left[M_{0}+\frac{K \beta}{1+\chi}\left(z-\frac{d}{2}\right)\right] \hat{k} }
\end{aligned}
$$

where, $\beta=\Delta T / d=\left(T_{L}-T_{U}\right) / d$ is the temperature gradient, $\hat{k}$ is the unit vector in the $z$-direction and the subscript $b$ denotes the basic state. It may be noted that the fluid and solid phases have the same temperatures at the bounding surfaces of the porous layer.

\subsection{The perturbed state}

To investigate the conditions under which the quiescent solution is stable against small disturbances, we consider a perturbed state in the form

$\vec{q}=\vec{q}^{\prime}, \quad p=p_{b}(z)+p^{\prime}, \quad T_{f}=T_{f b}(z)+T_{f}^{\prime}$,

$T_{s}=T_{s b}(z)+T_{s}^{\prime}, \quad \vec{H}=\vec{H}_{b}(z)+\vec{H}^{\prime}$,

$\vec{M}=\vec{M}_{b}(z)+\vec{M}^{\prime}$

where, $\vec{q}^{\prime}=\left(u^{\prime}, v^{\prime}, w^{\prime}\right), p^{\prime}, T_{f}^{\prime}, T_{s}^{\prime}, \vec{H}^{\prime}=\left(H_{x}^{\prime}, H_{y}^{\prime}, H_{z}^{\prime}\right)$ and $\vec{M}^{\prime}=\left(M_{x}^{\prime}, M_{y}^{\prime}, M_{z}^{\prime}\right)$ are perturbed variables and are assumed to be small.

Substituting Eq. (11) into Eqs. (7) and (8), and using Eqs. (6a), (6b), we obtain (after dropping the primes)

$$
\begin{aligned}
& H_{x}+M_{x}=\left(1+M_{0} / H_{0}\right) H_{x} \\
& H_{y}+M_{y}=\left(1+M_{0} / H_{0}\right) H_{y} \\
& H_{z}+M_{z}=(1+\chi) H_{z}-K T_{f} .
\end{aligned}
$$

Using Eq. (11) in Eq. (2), linearizing and taking curl on the resulting equation (after neglecting primes), the $z$-component can be written as

$$
\frac{\rho_{0}}{\varepsilon} \frac{\partial \zeta}{\partial t}=\tilde{\mu}_{f} \nabla^{2} \zeta-\frac{\mu_{f}}{k} \zeta+2 \frac{\rho_{0}}{\varepsilon} \Omega \frac{\partial w}{\partial z}
$$

which is the vorticity transport equation and $\zeta=$ $\partial v / \partial x-\partial u / \partial y$ is the $z$-component of vorticity.

Again substituting Eq. (11) into momentum Eq. (2), linearizing, eliminating the pressure term by taking 
curl twice and using Eq. (12) the $z$-component of the resulting equation can be obtained as (after dropping the primes):

$$
\begin{aligned}
\left(\frac{\rho_{0}}{\varepsilon}\right. & \left.\frac{\partial}{\partial t}+\frac{\mu_{f}}{k}-\tilde{\mu}_{f} \nabla^{2}\right) \nabla^{2} w \\
= & -2 \frac{\rho_{0}}{\varepsilon} \Omega \frac{\partial \zeta}{\partial z}-\mu_{0} K \beta \frac{\partial}{\partial z}\left(\nabla_{h}^{2} \phi\right) \\
& +\frac{\mu_{0} K^{2} \beta}{1+\chi} \nabla_{h}^{2} T_{f}+\rho_{0} \alpha_{t} g \nabla_{h}^{2} T_{f}
\end{aligned}
$$

where, $\nabla_{h}^{2}=\partial^{2} / \partial x^{2}+\partial^{2} / \partial y^{2}$ is the horizontal Laplacian operator.

Equations (3) and (4), after using Eq. (11) and linearizing, take the following form (after dropping the primes):

$$
\begin{aligned}
& \varepsilon\left(\rho_{0} c\right)_{f} \frac{\partial T_{f}}{\partial t}+\left(\rho_{0} c\right)_{f} w \frac{d T_{f b}}{d z} \\
& \quad=\varepsilon k_{f} \nabla^{2} T_{f}+h\left(T_{s}-T_{f}\right) \\
& (1-\varepsilon)\left(\rho_{0} c\right)_{s} \frac{\partial T_{s}}{\partial t}=(1-\varepsilon) k_{s} \nabla^{2} T_{s}-h\left(T_{s}-T_{f}\right) .
\end{aligned}
$$

Equations (6a), (6b), after substituting Eq. (11) and using Eq. (12), may be written as (after dropping the primes)

$$
\left(1+\frac{M_{0}}{H_{0}}\right) \nabla_{h}^{2} \phi+(1+\chi) \frac{\partial^{2} \phi}{\partial z^{2}}-K \frac{\partial T_{f}}{\partial z}=0 .
$$

\section{Linear stability theory}

To solve the above linear stability equations, we assume the normal mode expansion of the dependent variables in the form

$$
\begin{aligned}
\left\{w, T_{f}, T_{s}, \phi, \zeta\right\}= & \left\{W(z), \Theta_{f}(z), \Theta_{s}(z), \Phi(z), Z(z)\right\} \\
& \times \exp [i(\ell x+m y+\sigma t)]
\end{aligned}
$$

where, $\ell$ and $m$ are wave numbers in the $x$ and $y$ directions, respectively, $W(z)$ is the amplitude of vertical component of perturbed velocity, $\Theta_{f}(z)$ is the amplitude of perturbed fluid temperature, $\Theta_{S}(z)$ is the amplitude of perturbed solid temperature, $\Phi(z)$ is the amplitude of perturbed magnetic potential and $Z(z)$ is the amplitude of perturbed vertical component of vorticity and $\sigma$ is the growth rate which is complex, in general.
Substituting Eq. (18) into Eqs. (13)-(17), and nondimensionalizing the variables by setting

$$
\begin{aligned}
& \left(x^{*}, y^{*}, z^{*}\right)=\left(\frac{x}{d}, \frac{y}{d}, \frac{z}{d}\right), \quad t^{*}=\frac{\kappa_{f}}{d^{2}} t, \\
& W^{*}=\frac{d}{\varepsilon \kappa_{f}} W, \quad \Theta_{f}^{*}=\frac{1}{\beta d} \Theta_{f} \\
& \Theta_{s}^{*}=\frac{1}{\beta d} \Theta_{s}, \quad \Phi^{*}=\frac{(1+\chi)}{K \beta d^{2}} \Phi, \quad Z^{*}=\frac{d^{2}}{v} Z
\end{aligned}
$$

where $\kappa_{f}=k_{f} /\left(\rho_{0} c\right)_{f}$ is the effective thermal diffusivity of the fluid, we obtain (after dropping the asterisks for simplicity)

$$
\begin{aligned}
& {\left[\Lambda\left(D^{2}-a^{2}\right)-D a^{-1}-\frac{\sigma}{P r}\right]\left(D^{2}-a^{2}\right) W} \\
& =-a^{2} R\left[M_{1} D \Phi-\left(1+M_{1}\right) \Theta_{f}\right]+T a^{1 / 2} D Z \\
& \left(D^{2}-a^{2}-\sigma\right) \Theta_{f}+H_{t}\left(\Theta_{s}-\Theta_{f}\right)=-W \\
& \left(D^{2}-a^{2}-\alpha \sigma\right) \Theta_{s}+\gamma H_{t}\left(\Theta_{f}-\Theta_{s}\right)=0 \\
& \left(D^{2}-a^{2} M_{3}\right) \Phi-D \Theta_{f}=0 \\
& {\left[\Lambda\left(D^{2}-a^{2}\right)-D a^{-1}-\frac{\sigma}{P r}\right] Z=-T a^{1 / 2} D W .}
\end{aligned}
$$

Here, $D=d / d z$ is the differential operator, $a=$ $\sqrt{\ell^{2}+m^{2}}$ is the overall horizontal wave number, $R=$ $\rho_{0} \alpha_{t} g \beta d^{4} / \varepsilon \mu_{f} \kappa_{f}$ is the Rayleigh number and it is the ratio of buoyant to viscous forces, $T a=4 \Omega^{2} d^{4} / \nu^{2} \varepsilon^{2}$ is the Taylor number and it is the ratio of Coriolis to viscous forces, $\operatorname{Pr}=v / \varepsilon \kappa_{f}$ is the Prandtl number, $\Lambda=\tilde{\mu}_{f} / \mu_{f}$ is the ratio of viscosities, $D a=k / d^{2}$ is the Darcy number, $M_{1}=\mu_{0} K^{2} \beta /(1+\chi) \alpha_{t} \rho_{0} g$ is the magnetic number and it is the ratio of magnetic to gravitational forces, $M_{3}=\left(1+M_{0} / H_{0}\right) /(1+\chi)$ is the measure of nonlinearity of magnetization and $M_{3}=1$ corresponds to linear magnetization, $H_{t}=$ $h d^{2} / \varepsilon k_{f}$ is the scaled inter-phase heat transfer coefficient, $\alpha=\kappa_{f} / \kappa_{s}$ is the ratio of diffusivities, and $\gamma=\varepsilon k_{f} /(1-\varepsilon) k_{s}$ is the porosity modified conductivity ratio and $\sigma$ is the growth rate.

The boundaries are considered to be stress-free, ferromagnetic, and perfect conductors of heat. The corresponding boundary conditions are:

$$
\begin{aligned}
& W=0=D^{2} W, \quad \Theta_{f}=0=\Theta_{s}, \\
& D \Phi=0=D Z \quad \text { at } z=0,1 .
\end{aligned}
$$


Equations (20)-(24) admit solution satisfying the boundary conditions in the form

$W=A_{1} \sin \pi z, \quad \Theta_{f}=A_{2} \sin \pi z$,

$\Theta_{s}=A_{3} \sin \pi z$,

$\Phi=-\left(A_{4} / \pi\right) \cos \pi z, \quad Z=-\left(A_{5} / \pi\right) \cos \pi z$ where $A_{1}-A_{5}$ are constants. Substitution of (26) into (20)-(24) and eliminating the constants $A_{1}-A_{5}$ from the resulting equations yields the following characteristic equation:

$$
\left|\begin{array}{ccccc}
\delta^{2}\left(\frac{\sigma}{P r}+\Lambda \delta^{2}+D a^{-1}\right) & -a^{2} R\left(1+M_{1}\right) & 0 & a^{2} R M_{1} & -\sqrt{T a} \\
-1 & \left(\alpha \sigma+\delta^{2}+H_{t}\right) & -H_{t} & 0 & 0 \\
0 & -\gamma H_{t} & \left(\sigma+\delta^{2}+\gamma H_{t}\right) & 0 & 0 \\
0 & \pi^{2} & 0 & -\left(\pi^{2}+M_{3} a^{2}\right) & 0 \\
\pi^{2} \sqrt{T a} & 0 & 0 & 0 & \left(\frac{\sigma}{P r}+\Lambda \delta^{2}+D a^{-1}\right)
\end{array}\right|=0
$$

where, $\delta^{2}=\pi^{2}+a^{2}$. Expanding the above determinant gives the following expression for the Rayleigh number $R$ :

$R=\frac{\left[\begin{array}{l}\left(\pi^{2}+M_{3} a^{2}\right)\left\{\pi^{2} \operatorname{Pr}^{2} T a+\delta^{2}\left(D a^{-1} \operatorname{Pr}+\operatorname{Pr} \delta^{2} \Lambda+\sigma\right)^{2}\right\} \times \\ \left\{\left(\delta^{2}+\sigma\right)\left(\delta^{2}+\sigma \alpha\right)+H_{t}(1+\gamma) \delta^{2}+H_{t}(\alpha+\gamma) \sigma\right\}\end{array}\right]}{\operatorname{Pra}^{2}\left\{\pi^{2}+M_{3}\left(1+M_{1}\right) a^{2}\right\}\left(\delta^{2}+\gamma H_{t}+\alpha \sigma\right)\left(D a^{-1} \operatorname{Pr}+\operatorname{Pr} \delta^{2} \Lambda+\sigma\right)}$.

To examine the stability of the system, the real part of $\sigma$ is set to zero and we take $\sigma=i \omega$. Substituting $\sigma=i \omega$ in (28) and clearing the complex quantities from the denominator, we get

$R=\frac{\left(\pi^{2}+M_{3} a^{2}\right)}{\operatorname{Pra}^{2}\left\{\pi^{2}+M_{3}\left(1+M_{1}\right) a^{2}\right\}\left\{\left(\delta^{2}+\gamma H_{t}\right)^{2}+\alpha^{2} \omega^{2}\right\}\left\{\operatorname{Pr}^{2}\left(D a^{-1}+\delta^{2} \Lambda\right)^{2}+\omega^{2}\right\}}\left(\Delta_{1}+i \omega \Delta_{2}\right)$

where the expressions for $\Delta_{1}$ and $\Delta_{2}$ are given in the Appendix. Since the Rayleigh number $R$ is a physical quantity, it must be real. Hence, from (29) it implies either $\omega=0$ or $\Delta_{2}=0(\omega \neq 0)$, and accordingly the condition for the onset of stationary and oscillatory convection is obtained.

\subsection{Stationary convection $(\omega=0)$}

The stationary convection occurs at $R=R^{s}$, where

$R^{s}=\frac{\left(\pi^{2}+M_{3} a^{2}\right) \delta^{2}\left\{\pi^{2} T a+\delta^{2}\left(D a^{-1}+\delta^{2} \Lambda\right)^{2}\right\}\left\{H_{t}(1+\gamma)+\delta^{2}\right\}}{\left\{\pi^{2}+M_{3}\left(1+M_{1}\right) a^{2}\right\} a^{2}\left(\delta^{2}+\gamma H_{t}\right)\left(D a^{-1}+\delta^{2} \Lambda\right)}$.

The critical Rayleigh number $R_{c}^{s}$ and the corresponding critical wave number $a_{c}$ are determined numerically for various values of $T a, D a, \Lambda, M_{1}, M_{3}, \gamma$ and $H_{t}$ because the expression for the Rayleigh number is complicated for analytical treatment. Equation (30) coincides with the one obtained by Sunil et al. [29]. It is also interesting to check (30) under some limiting cases. When $M_{1}=0$ (i.e., ordinary viscous fluid case),
(30) reduces to

$$
R^{s}=\frac{\delta^{2}\left\{\delta^{2}+H_{t}(1+\gamma)\right\}\left\{\pi^{2} T a+\delta^{2}\left(D a^{-1}+\delta^{2} \Lambda\right)^{2}\right\}}{a^{2}\left(\delta^{2}+\gamma H_{t}\right)\left(D a^{-1}+\delta^{2} \Lambda\right)} .
$$

The above expression coincides with those of Straughan [27] and Malashetty et al. [28] when $\Lambda=0$ 
and after rescaling the Rayleigh and Taylor numbers with the Darcy number. When $T a=0$, (30) reduces to

$R^{s}=\frac{\delta^{4}\left(D a^{-1}+\delta^{2} \Lambda\right)\left\{H_{t}(1+\gamma)+\delta^{2}\right\}\left(\pi^{2}+M_{3} a^{2}\right)}{a^{2}\left\{\pi^{2}+M_{3}\left(1+M_{1}\right) a^{2}\right\}\left(\delta^{2}+\gamma H_{t}\right)}$

which coincides with the expression obtained by Shivakumara et al. [20].

When $H_{t}=0$ (LTE case) and $M_{1}=0$, (30) reduces to

$R^{s}=\frac{\delta^{2}\left\{\pi^{2} T a+\delta^{2}\left(D a^{-1}+\delta^{2} \Lambda\right)^{2}\right\}}{a^{2}\left(D a^{-1}+\delta^{2} \Lambda\right)}$

which coincides with the one given by Shivakumara et al. [31].

When $H_{t} \rightarrow \infty$, (30) reduces to

$$
\begin{aligned}
R^{s}\left(\frac{\gamma}{1+\gamma}\right)= & \frac{\rho_{0} \alpha_{t} g \beta d^{4}}{\left\{\varepsilon \kappa_{f}+(1-\varepsilon) \kappa_{s}\right\} \mu} \\
= & R_{M p}^{s}=\frac{\delta^{2}\left\{\pi^{2} T a+\delta^{2}\left(D a^{-1}+\delta^{2} \Lambda\right)^{2}\right\}}{a^{2}\left(D a^{-1}+\delta^{2} \Lambda\right)} \\
& \times \frac{\left(\pi^{2}+M_{3} a^{2}\right)}{\left\{\pi^{2}+M_{3}\left(1+M_{1}\right) a^{2}\right\}}
\end{aligned}
$$

Here, it may be noted that the expression for the Rayleigh number is based on the mean properties of the porous medium and coincides for the LTE case. When $M_{1}=0=\Lambda$, then the above equation coincides with the classical result obtained by Palm and Tyvand [32] for the problem of thermal instability in a rotating viscous fluid saturated porous layer. For very large $M_{1}$, the results for the magnetic mechanism alone operating in the absence of buoyancy effects is obtained. The corresponding magnetic Rayleigh number $R_{m}\left(=R^{s} M_{1}\right)$ is expressed as follows:

$$
\begin{aligned}
R_{m}= & R^{s} M_{1} \\
= & \frac{\delta^{2}\left\{\delta^{2}+H_{t}(1+\gamma)\right\}\left\{\pi^{2} T a+\delta^{2}\left(D a^{-1}+\delta^{2} \Lambda\right)^{2}\right\}}{a^{2}\left(\delta^{2}+\gamma H_{t}\right)\left(D a^{-1}+\delta^{2} \Lambda\right)} \\
& \times \frac{\left(\pi^{2}+M_{3} a^{2}\right)}{M_{3} a^{2}} .
\end{aligned}
$$

In addition, when $M_{3} \rightarrow \infty$, we note that (35) turns out to be the same as (31) (i.e., $R_{m}=R^{s}$ ).

\subsection{Oscillatory convection $(\omega \neq 0)$}

The oscillatory onset corresponds to $\Delta_{2}=0(\omega \neq 0)$ in (29) and this gives a dispersion relation of the form

$A_{1}\left(\omega_{i}^{2}\right)^{2}+A_{2}\left(\omega_{i}^{2}\right)+A_{3}=0$

where,

$$
\begin{aligned}
& A_{1}=\alpha^{2} \delta^{2}\left\{H_{t}+D a^{-1} \operatorname{Pr}+\delta^{2}(1+\operatorname{Pr} \Lambda)\right\} \\
& A_{2}=\left(D a^{-1}\right)^{3} \operatorname{Pr}^{3} \alpha^{2} \delta^{2}+H_{t}^{2} \gamma \delta^{4}(1+\gamma \\
& +\operatorname{Pr} \alpha \Lambda+\operatorname{Pr} \gamma \Lambda)+\left(D a^{-1}\right)^{2} \operatorname{Pr}^{2} \alpha^{2} \delta^{2} \\
& \times\left\{H_{t}+\delta^{2}(1+3 \operatorname{Pr} \Lambda)\right\} \\
& +\delta^{2}\left\{\pi^{2} \operatorname{Pr}^{2} \alpha^{2} \operatorname{Ta}(\operatorname{Pr} \Lambda-1)\right. \\
& \left.+\delta^{6}(1+\operatorname{Pr} \Lambda)\left(1+\operatorname{Pr}^{2} \alpha^{2} \Lambda^{2}\right)\right\} \\
& +H_{t}\left\{\delta^{6}\left(1+2 \gamma+2 \operatorname{Pr} \Lambda \gamma+\operatorname{Pr}^{2} \alpha^{2} \Lambda^{2}\right)\right. \\
& \left.-\pi^{2} \operatorname{Pr} \alpha^{2} T a\right\} \\
& +D a^{-1}\left\{\begin{array}{l}
\pi^{2} \operatorname{Pr}^{2} \alpha^{2} T a+\delta^{2}\left(H_{t}^{2} \operatorname{Pr} \Lambda(\alpha+\gamma)\right) \\
+2 H_{t} \operatorname{Pr} \delta^{4}\left(\gamma+\operatorname{Pr} \alpha^{2} \Lambda\right) \\
+\delta^{6}\left(1+2 \operatorname{Pr} \alpha^{2} \Lambda+3 \operatorname{Pr}^{2} \alpha^{2} \Lambda^{2}\right)
\end{array}\right\} \\
& A_{3}=\left(D a^{-1}\right)^{3} \operatorname{Pr}^{2} \delta^{2}\left\{H_{t}^{2} \gamma \operatorname{Pr}(\alpha+\gamma)+2 H_{t} \gamma \delta^{2}\right. \\
& \left.+\delta^{4}\right\}+\left(D a^{-1}\right)^{2} \operatorname{Pr}^{2} \delta^{8}(1+3 \operatorname{Pr} \Lambda) \\
& +\left(D a^{-1}\right)^{2} \operatorname{Pr}^{2} \delta^{4}\left\{H_{t}^{2} \gamma(1+\gamma+3 \operatorname{Pr} \alpha \Lambda\right. \\
& \left.+3 \operatorname{Pr} \gamma \Lambda)+H_{t} \delta^{2}(1+2 \gamma+6 \operatorname{Pr} \gamma \Lambda)\right\} \\
& +D a^{-1} \operatorname{Pr}^{2}\left\{\delta^{4} \pi^{2} \operatorname{PrTa}+\delta^{10} \Lambda(2+3 \operatorname{Pr} \Lambda)\right. \\
& \left.+2 H_{t}\left\{\pi^{2} \operatorname{Pr} T a \delta^{2}+\delta^{8} \Lambda(1+2 \gamma+3 \operatorname{Pr} \Lambda \gamma)\right\}\right\} \\
& +D a^{-1} \operatorname{Pr}^{2} H_{t}^{2} \gamma\left\{\pi^{2} \operatorname{PrTa}(\alpha+\gamma)\right. \\
& \left.+\delta^{6} \Lambda(2+2 \gamma+3 \operatorname{Pr} \alpha \Lambda+3 \operatorname{Pr} \gamma \Lambda)\right\} \\
& +\delta^{6}\left\{\pi^{2} \alpha^{2} \operatorname{Ta}(\operatorname{Pr} \Lambda-1)\right\} \\
& +\delta^{8} \Lambda^{2} \operatorname{Pr}^{2}(1+\operatorname{Pr} \Lambda) \\
& +\delta^{2} H_{t}^{2} \gamma\left\{\pi^{2} \operatorname{Ta}(\gamma(\operatorname{Pr} \Lambda-1)-1+\operatorname{Pr} \gamma \Lambda)\right. \\
& \left.+\delta^{6} \Lambda^{2}(1+\gamma+\operatorname{Pr} \alpha \Lambda+\operatorname{Pr} \gamma \Lambda)\right\} \\
& +H_{t} \delta^{2} \operatorname{Pr}^{2}\left\{\pi^{2} \operatorname{Ta}(2 \gamma(\operatorname{Pr} \Lambda-1)-1)\right. \\
& \left.+\delta^{6} \Lambda^{2}(1+2 \gamma(1+\operatorname{Pr} \Lambda))\right\} \text {. }
\end{aligned}
$$

The oscillatory convection occurs at $R=R^{o}$, where 
$R^{o}=\frac{\left(\pi^{2}+M_{3} a^{2}\right) \Delta_{1}}{\operatorname{Pra}^{2}\left\{\pi^{2}+M_{3}\left(1+M_{1}\right) a^{2}\right\}\left\{\left(\delta^{2}+\gamma H_{t}\right)^{2}+\alpha^{2} \omega^{2}\right\}\left\{\operatorname{Pr}^{2}\left(D a^{-1}+\delta^{2} \Lambda\right)^{2}+\omega^{2}\right\}}$

and $\omega^{2}$ is given by (36). For the existence of oscillatory convection, $\omega_{i}^{2}$ should be positive. From (36) it can be seen that the occurrence of oscillatory convection depends on the values of various physical parameters involved therein. In the absence of rotation (i.e., $T a=0$ ), a glance at (36) reveals that $\omega_{i}^{2}$ will not have any positive roots since $A_{i}>0(i=1,2,3)$. Thus the occurrence of oscillatory convection does not depend on LTE or LTNE model but it is only due to the presence of Coriolis force due to rotation. For the LTE model in the presence of rotation, it is shown that oscillatory convection is not a preferred mode of instability unless the value of the Prandtl number is less than unity and the Taylor number exceeds a threshold value (Shivakumara [26]). Under the circumstances, it is obvious to reason out that for the problem under investigation oscillatory convection will be a preferred mode of instability only if the Prandtl number is less than unity. Since the Prandtl number is greater than unity for ferromagnetic fluids (whether they are water based or any other organic liquid based), oscillatory convection is not a preferred mode of instability and hence we have limited our discussion to only stationary onset.

\section{Asymptotic values of $\boldsymbol{R}^{s}$ for small and large values of $\boldsymbol{H}_{t}$}

An asymptotic analysis is being carried out to obtain an expression for the Rayleigh number $R^{s}$ and the corresponding wave number for small as well as large values of $H_{t}$.

\subsection{Case 1: $H_{t} \ll 1$}

For this case, the Rayleigh number $R^{s}$ is slightly above the corresponding value for the LTE case. Accordingly, we expand $R^{S}$ given by (30) in a power series in $H_{t}$ as

$$
\begin{aligned}
R^{s}= & \frac{\left(\pi^{2}+a^{2}\right)\left\{\pi^{2} T a+\left(\pi^{2}+a^{2}\right)\left(D a^{-1}+\left(\pi^{2}+a^{2}\right) \Lambda\right)^{2}\right\}\left(\pi^{2}+M_{3} a^{2}\right)}{a^{2}\left\{D a^{-1}+\left(\pi^{2}+a^{2}\right) \Lambda\right\}\left\{\pi^{2}+M_{3}\left(1+M_{1}\right) a^{2}\right\}} \\
& \times\left[1+\frac{H_{t}}{\left(\pi^{2}+a^{2}\right)}-\frac{\gamma H_{t}^{2}}{\left(\pi^{2}+a^{2}\right)^{2}}+\cdots\right]
\end{aligned}
$$

To minimize $R^{s}$ up to $O\left(H_{t}^{2}\right)$, we set $\partial R^{s} / \partial a=0$ and obtain an expression of the form

$$
B_{1} \gamma H_{t}^{2}+B_{2} H_{t}+B_{3} \cdots=0
$$

where $B_{1}, B_{2}$ and $B_{3}$ are lengthy expressions and they are not given here.

We also expand $a$ in power series of $H_{t}$ as

$a=a_{0}+a_{1} H_{t}+a_{2} H_{t}^{2}+\cdots$

where, $a_{0}$ is the critical wave number for the LTE case. Substituting (40) into (39) and equating the coefficients of like powers of $H_{t}$, we find $a_{1}$ and $a_{2}$ and are given by

$a_{1}=\frac{\Delta_{1}^{\prime}}{\Delta^{\prime}}, \quad a_{2}=\frac{\Delta_{2}^{\prime}}{\Delta^{\prime}}$

where $\Delta^{\prime}, \Delta_{1}^{\prime}$ and $\Delta_{2}^{\prime}$ are lengthy expressions and they are not given here but these expressions are used during numerical calculations. With the values of $a_{0}, a_{1}$ and $a_{2}$, (40) gives the critical wave number and consequently using this in (38) one can obtain the critical Rayleigh number for small $H_{t}$. 
Table 1 Comparison of the asymptotic (A) and exact (E) values of the critical Rayleigh number $\left(R_{c}^{s}\right)$ and the critical wave number $\left(a_{c}\right)$ for different values of $\log _{10} H_{t}$ with $M_{1}=1, M_{3}=1, D a^{-1}=100, T a=100, \Lambda=1$ and $\gamma=1$

\begin{tabular}{cllll}
\hline $\log _{10} H_{t}$ & $R_{c}^{s}(A)$ & $a_{c}(A)$ & $R_{c}^{s}(E)$ & $a_{c}(E)$ \\
\hline-2.0 & 3144.52 & 3.366 & 3144.51 & 3.372 \\
-1.0 & 3157.75 & 3.373 & 3157.74 & 3.378 \\
0.0 & 3282.81 & 3.426 & 3283.09 & 3.431 \\
1.0 & 3963.00 & 3.098 & 4122.12 & 3.630 \\
2.0 & 5754.34 & 3.488 & 5726.71 & 3.495 \\
3.0 & 6220.59 & 3.381 & 6220.55 & 3.386 \\
4.0 & 6279.42 & 3.368 & 6279.4 & 3.373 \\
5.0 & 6285.41 & 3.366 & 6285.39 & 3.371 \\
\hline
\end{tabular}

4.2 Case 2: $H_{t} \gg 1$

For this case, the Rayleigh number takes the form

$$
\begin{aligned}
R^{s}= & \frac{\left(\pi^{2}+a^{2}\right)\left\{\pi^{2} T a+\left(\pi^{2}+a^{2}\right)\left(D a^{-1}+\left(\pi^{2}+a^{2}\right) \Lambda\right)^{2}\right\}\left(\pi^{2}+M_{3} a^{2}\right)}{a^{2}\left(D a^{-1}+\left(\pi^{2}+a^{2}\right) \Lambda\right)\left\{\pi^{2}+M_{3}\left(1+M_{1}\right) a^{2}\right\}} \\
& \times\left(\frac{1+\gamma}{\gamma}\right)\left[1-\frac{\left(\pi^{2}+a^{2}\right)}{\gamma(1+\gamma) H_{t}}+\frac{\left(\pi^{2}+a^{2}\right)^{2}}{\gamma^{2}(1+\gamma) H_{t}^{2}}+\cdots\right]
\end{aligned}
$$

We minimize this with respect to $a$ in a similar way as we did in the small $H_{t}$ case and obtain the following expression:

$C_{1} \frac{1}{\gamma^{3} H_{t}^{2}}+C_{2} \frac{1}{\gamma^{2} H_{t}}+C_{3} \frac{1+\gamma}{\gamma}+\cdots=0$

where $C_{1}, C_{2}$ and $C_{3}$ are lengthy expressions and not presented here.

Similarly, we expand $a$ in the form

$a=a_{0}+\frac{a_{1}^{\prime}}{H_{t}}+\frac{a_{2}^{\prime}}{H_{t}^{2}}+\cdots$

where $a_{0}$ is the critical wave number for the LTE case and $a_{1}^{\prime}$ and $a_{2}^{\prime}$ are to be determined. Substituting (44) into (43) and equating the coefficients of like powers of $H_{t}$, we find $a_{1}^{\prime}$ and $a_{2}^{\prime}$ and are given by

$a_{1}^{\prime}=\frac{\Delta_{1}^{\prime \prime}}{\Delta^{\prime \prime}}, \quad a_{2}^{\prime}=\frac{\Delta_{2}^{\prime \prime}}{\Delta^{\prime \prime}}$

where, $\Delta^{\prime \prime} \Delta_{1}^{\prime \prime}$ and $\Delta_{2}^{\prime \prime}$ are quite lengthy expressions and they are not given here. But they are used during numerical calculations of the critical wave number.
Again with the values of $a_{0}, a_{1}^{\prime}$ and $a_{2}^{\prime}$, we compute the critical wave number $a_{c}$ from (44) and finally using this value of $a_{c}$ one can obtain the critical Rayleigh number from (42) for large $H_{t}$.

The critical Rayleigh number $R_{c}^{s}$ (i.e., the critical value of $R^{s}$ obtained with respect to the wave number $a$ ) and the corresponding critical wave number $a_{c}$ are obtained for both small as well as large $H_{t}$ using the asymptotic formulae. The critical stability parameters $\left(R_{c}^{s}, a_{c}\right)$ so obtained are compared with the exact values obtained from (30) in Table 1 for representative values of $M_{1}=1, M_{3}=1, D a^{-1}=100=T a$, $\Lambda=1$ with $\gamma=1$. From the table, it is observed that an increasingly good agreement amongst the critical stability parameters as the value of $H_{t}$ either decreases or increases. The asymptotic (A) and exact (E) critical Darcy-Rayleigh $\left(R_{D c}^{s}=R_{c}^{s} D a\right)$ and the corresponding wave numbers obtained under the limiting case of an ordinary viscous fluid (i.e., $M_{1}=0$, $M_{3}=0$ ) saturating a densely packed (i.e., $\Lambda=0$ ) rotating porous layer for two values of Darcy-Taylor number $T a_{D}=0$ and 25 with $\gamma=1$ are compared with those of Malashetty et al. [28] in Table 2. It may be 
Table 2 Comparison of the asymptotic (A) and exact (E) values of the critical Darcy-Rayleigh number $\left(R_{D c}^{s}\right)$ and the critical wave number $\left(a_{c}\right)$ for different values of $\log _{10} H_{t}$ with $\gamma=1, M_{1}=0, M_{3}=0, \Lambda=0$ and $T a_{D}=0$ and 25

\begin{tabular}{|c|c|c|c|c|c|c|c|c|c|}
\hline \multirow[t]{2}{*}{$T a_{D}$} & \multirow[t]{2}{*}{$\log _{10} H_{t}$} & \multicolumn{4}{|c|}{ Malashetty et al. [28] } & \multicolumn{4}{|c|}{ Present analysis } \\
\hline & & $R_{c}^{s}(A)$ & $a_{c}(A)$ & $R_{c}^{s}(E)$ & $a_{c}(E)$ & $R_{c}^{s}(A)$ & $a_{c}(A)$ & $R_{c}^{s}(E)$ & $a_{c}(E)$ \\
\hline \multirow[t]{8}{*}{0} & -2.0 & 39.498 & 3.142 & 39.498 & 3.142 & 39.498 & 3.142 & 39.498 & 3.142 \\
\hline & -1.0 & 39.677 & 3.149 & 39.677 & 3.149 & 39.677 & 3.149 & 39.677 & 3.149 \\
\hline & 0.0 & 41.356 & 3.211 & 41.362 & 3.211 & 41.358 & 3.210 & 41.362 & 3.211 \\
\hline & 1.0 & 49.346 & 3.131 & 52.360 & 3.436 & 49.752 & 2.829 & 52.360 & 3.436 \\
\hline & 2.0 & 72.622 & 3.250 & 72.340 & 3.271 & 72.625 & 3.270 & 72.340 & 3.271 \\
\hline & 3.0 & 78.191 & 3.157 & 78.191 & 3.157 & 78.191 & 3.157 & 78.191 & 3.157 \\
\hline & 4.0 & 78.879 & 3.143 & 78.879 & 3.143 & 78.879 & 3.143 & 78.879 & 3.143 \\
\hline & 5.0 & 78.949 & 3.142 & 78.949 & 3.142 & 78.949 & 3.142 & 78.949 & 3.142 \\
\hline \multirow[t]{8}{*}{25} & -2.0 & 397.191 & 7.096 & 397.191 & 7.096 & 397.191 & 7.096 & 397.191 & 7.096 \\
\hline & -1.0 & 367.738 & 7.112 & 367.738 & 7.112 & 367.738 & 7.112 & 367.738 & 7.112 \\
\hline & 0.0 & 373.012 & 7.262 & 373.014 & 7.262 & 373.012 & 7.261 & 373.014 & 7.262 \\
\hline & 1.0 & 414.289 & 8.141 & 414.019 & 8.198 & 414.416 & 7.611 & 414.019 & 8.198 \\
\hline & 2.0 & 644.433 & 6.744 & 576.92 & 9.280 & 644.02 & 6.145 & 576.92 & 9.280 \\
\hline & 3.0 & 712.760 & 7.421 & 712.665 & 7.426 & 712.760 & 7.428 & 712.665 & 7.426 \\
\hline & 4.0 & 732.055 & 7.127 & 732.055 & 7.127 & 732.055 & 7.127 & 732.055 & 7.127 \\
\hline & 5.0 & 734.039 & 7.097 & 734.039 & 7.097 & 734.039 & 7.097 & 734.039 & 7.097 \\
\hline
\end{tabular}

seen that the results presented for different values of $\log _{10} H_{t}$ agree very well with each other.

\section{Results and discussion}

The combined effect of LTNE and Coriolis force due to rotation on thermal convection in a ferromagnetic fluid saturated horizontal Brinkman porous layer in the presence of a uniform vertical magnetic field is investigated. The condition for the occurrence of stationary and oscillatory onset is established analytically. It is observed that oscillatory convection is not a preferred mode of instability and the results are discussed for stationary convection. By performing careful numerical calculations it is shown that the rotating ferrofluid saturated porous layer is capable of supporting some new results which are not found in the previous investigation.

From (30), we note that $\partial R^{s} / \partial T a>0, \partial R^{s} / \partial M_{1}<$ 0 and $\partial R^{s} / \partial M_{3}<0$. Hence, the effect of increasing $T a$ is to delay and increasing $M_{1}$ as well as $M_{3}$ is to hasten the onset of ferromagnetic convection. Further, it is observed that

$$
\begin{aligned}
\frac{\partial R^{s}}{\partial D a^{-1}}= & \frac{\left(a^{2} M_{3}+\pi^{2}\right)\left\{H_{t}(1+\gamma)+\delta^{2}\right\} \delta^{2}}{a^{2}\left\{a^{2}\left(1+M_{1}\right) M_{3}+\pi^{2}\right\}\left(H_{t} \gamma+\delta^{2}\right)} \\
& \times\left[2 \delta^{2}-\frac{\pi^{2} T a+\delta^{2}\left(D a^{-1}+\delta^{2} \Lambda\right)^{2}}{\left(D a^{-1}+\delta^{2} \Lambda\right)^{2}}\right] \\
\frac{\partial R^{s}}{\partial \Lambda}= & \frac{\left(a^{2} M_{3}+\pi^{2}\right)\left\{H(1+\gamma)+\delta^{2}\right\} \delta^{4}}{a^{2}\left\{a^{2}\left(1+M_{1}\right) M_{3}+\pi^{2}\right\}\left(H \gamma+\delta^{2}\right)} \\
& \times\left[2 \delta^{2}-\frac{\pi^{2} T a+\delta^{2}\left(D a^{-1}+\delta^{2} \Lambda\right)^{2}}{\left(D a^{-1}+\delta^{2} \Lambda\right)^{2}}\right] .
\end{aligned}
$$

When $T a=0$, from the above equations it is seen that $R^{s}$ is an increasing function of $D a^{-1}$ and $\Lambda$ indicating their effect is to stabilize the system. When $T a \neq 0$, however, it is observed that the right-hand side of (46) and (47) may be either negative or positive depending on the parametric values. That is to say that an increase in the value of $D a^{-1}$ and $\Lambda$ might lead to instability of a rotating ferromagnetic fluid saturated porous layer.

To visualize this behavior, the variation of $R_{c}^{s}$ as a function of $D a^{-1}$ is shown in Fig. 2 for different values of $\Lambda$ when $T a$ is fixed at $10^{6}$ for $M_{1}=1, M_{3}=1$, 


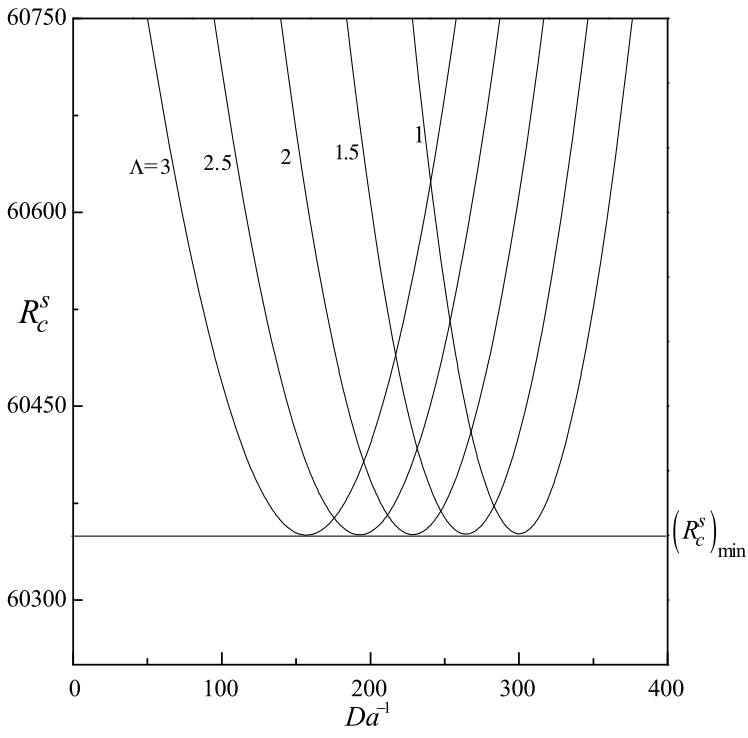

Fig. 2 Variation of $R_{c}^{s}$ with $D a^{-1}$ for different values of $\Lambda$ with $T a=10^{6}, M_{1}=1, M_{3}=1, \gamma=0.5$ and $H_{t}=100$

$\gamma=0.5$ and $H_{t}=100$. From the figure, it is observed that the destabilization due to $D a^{-1}$ manifests itself as minimum in the $R_{c}^{s}-D a^{-1}$ curve. The range of $D a^{-1}$ up to which the system becomes destabilized decreases with an increase in the value of $\Lambda$. This may be due to a delicate balance between the Coriolis and Darcy frictional forces, while elsewhere a strong 'two-dimensionality' prevails, being provided at lower values of $D a^{-1}$ by Coriolis forces, and at higher values of $D a^{-1}$ by frictional forces. This phenomenon is similar to the one observed by Chandrasekhar [33] in the study of thermal convective instability in a rotating fluid layer in the presence of vertical magnetic field; where it is observed that rotation/magnetic field destabilizes the system although their individual effect is to make the system more stable. Moreover, it is found that $R_{c}^{s}$ attains its minimum value with $D a^{-1}$, denoted by $\left(R_{c}^{s}\right)_{\min }$, at $D a^{-1}=D a_{m}^{-1}$, where

$D a_{m}^{-1}=\frac{\pi \sqrt{T a} \sqrt{\pi^{2}+a_{c}^{2}}-a_{c}^{4} \Lambda\left(\pi^{4}+1\right)-2 \pi^{2} a_{c}^{2} \Lambda}{\pi^{2}+a_{c}^{2}}$.

It is evident that $D a_{m}^{-1}$ decreases with an increase in the value of $\Lambda$ but increases with an increase in the value of $T a$. The above expression is same as the one obtained for an ordinary viscous fluid case and also independent of $H_{t}$ and $\gamma$ explicitly. The numerically

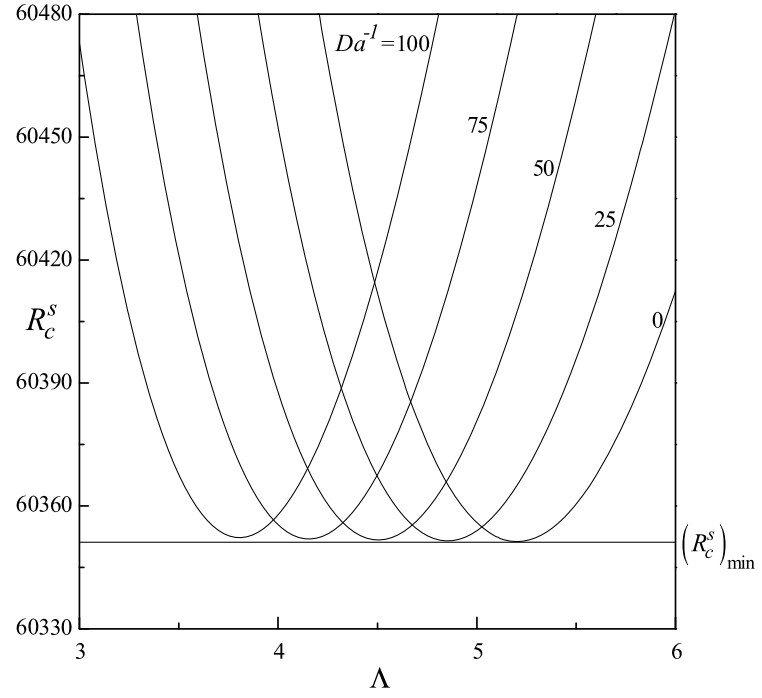

Fig. 3 Variation of $R_{c}^{s}$ with $\Lambda$ for different values of $D a^{-1}$ with $T a=10^{6}, M_{1}=1, M_{3}=1, \gamma=0.5$ and $H_{t}=100$

calculated values of $\left(R_{c}^{S}\right)_{\min }$ and $D a_{m}^{-1}$ are tabulated in Tables $3 \mathrm{a}, 3 \mathrm{~b}$ for different values of $\Lambda$ when $M_{1}=1$, $M_{3}=1, H_{t}=100,0$ (LTE case) and $\gamma=0.5$ for two values of $T a=10^{6}$ (see Table 3a) and $2 \times 10^{6}$ (see Table $3 \mathrm{~b})$. From the tables, it is seen that increasing $\Lambda$ is to decrease $D a_{m}^{-1}$, while increase in $T a$ is to increase the value of $D a_{m}^{-1}$. An interesting and important point to be noted here is that there is a coupling between the values of $\Lambda$ and $D a_{m}^{-1}$ such that $\left(R_{c}^{s}\right)_{\min }$ remains unaltered for a fixed value of $T a$ and also increase in the value of $T a$ is to increase the value of $\left(R_{c}^{s}\right)_{\min }$. That is, rotation has a stabilizing effect on the system.

A similar type of behavior is observed by varying $\Lambda$ and the results are presented in Fig. 3. The dual role of viscosity ratio $\Lambda$ on the onset of ferromagnetic convection in a rotating ferromagnetic fluid saturated porous layer is evident from this figure, where we note that $R_{c}^{s}$ passes through a minimum with an increase in the value of $\Lambda$. In this case, $R_{c}^{s}$ attains its minimum value with $\Lambda$ (i.e., $\left.\left(R_{c}^{s}\right)_{\min }\right)$ at $\Lambda=\Lambda_{m}$, where

$\Lambda_{m}=-\frac{D a^{-1}}{\left(\pi^{2}+a_{c}^{2}\right)}+\frac{\pi \sqrt{T a}}{\left(\pi^{2}+a_{c}^{2}\right) \sqrt{\left(\pi^{2}+a_{c}^{2}\right)}}$.

From the above equation, it is noted that $\Lambda_{m}$ decreases with an increase in the value of $D a^{-1}$, while it increases with increasing $T a$. The numerically calculated values of $\Lambda_{m}$ and the corresponding $\left(R_{c}^{s}\right)_{\min }$ for different values of $D a^{-1}$ when $\gamma=0.5, H_{t}=100,0$ (LTE case), $M_{1}=1$ and $M_{3}=1$ for two values of 
Table 3a Values of $\left(R_{c}^{s}\right)_{\min },\left(R_{m c}\right)_{\min }$ and $D a_{m}^{-1}$ for various vales of $\Lambda$ and $M_{1}$ when $M_{3}=1, \gamma=0.5$ and $T a=10^{6}$

\begin{tabular}{|c|c|c|c|c|c|c|c|}
\hline \multirow[t]{2}{*}{$H_{t}$} & \multirow[t]{2}{*}{$\Lambda$} & \multicolumn{2}{|c|}{$\begin{array}{l}\text { Simultaneous presence of } \\
\text { buoyancy and magnetic forces } \\
\left(M_{1}=1\right)\end{array}$} & \multicolumn{2}{|c|}{$\begin{array}{l}\text { Buoyancy forces alone present } \\
\left(M_{1}=0\right)\end{array}$} & \multicolumn{2}{|c|}{$\begin{array}{l}\text { Magnetic forces alone present } \\
\left(M_{1} \rightarrow \infty\right)\end{array}$} \\
\hline & & $\overline{\left(R_{c}^{s}\right)_{\min }}$ & $D a_{m}^{-1}$ & $\left(R_{c}^{s}\right)_{\min }$ & $D a_{m}^{-1}$ & $\overline{\left(R_{m c}\right)_{\min }}$ & $D a_{m}^{-1}$ \\
\hline \multirow[t]{5}{*}{100} & 1 & 60349.023 & 300.08 & 110707.454 & 398.773 & 128860.00 & 227.995 \\
\hline & 1.5 & 60349.023 & 264.34 & 110707.454 & 374.18 & 128860.00 & 180.62 \\
\hline & 2 & 60349.023 & 228.59 & 110707.454 & 349.588 & 128860.00 & 133.245 \\
\hline & 2.5 & 60349.023 & 192.85 & 110707.454 & 324.996 & 128860.00 & 85.87 \\
\hline & 3 & 60349.023 & 157.11 & 110707.454 & 300.404 & 128860.00 & 38.495 \\
\hline \multirow[t]{5}{*}{0} & 1 & 30065.000 & 471.590 & 51284.000 & 547.741 & 68966.00 & 397.866 \\
\hline & 1.5 & 30065.000 & 452.587 & 51284.000 & 532.937 & 68966.00 & 373.192 \\
\hline & 2 & 30065.000 & 433.584 & 51284.000 & 518.133 & 68966.00 & 348.518 \\
\hline & 2.5 & 30065.000 & 414.581 & 51284.000 & 503.328 & 68966.00 & 323.844 \\
\hline & 3 & 30065.000 & 395.578 & 51284.000 & 488.524 & 68966.00 & 299.170 \\
\hline
\end{tabular}

Table 3b Values of $\left(R_{c}^{s}\right)_{\min },\left(R_{m c}\right)_{\min }$ and $D a_{m}^{-1}$ for various vales of $\Lambda$ and $M_{1}$ when $M_{3}=1, \gamma=0.5$ and $T a=2 \times 10^{6}$

\begin{tabular}{|c|c|c|c|c|c|c|c|}
\hline \multirow[t]{2}{*}{$H_{t}$} & \multirow[t]{2}{*}{$\Lambda$} & \multicolumn{2}{|c|}{$\begin{array}{l}\text { Simultaneous presence of } \\
\text { buoyancy and magnetic forces } \\
\left(M_{1}=1\right)\end{array}$} & \multicolumn{2}{|c|}{$\begin{array}{l}\text { Buoyancy forces alone present } \\
\left(M_{1}=0\right)\end{array}$} & \multicolumn{2}{|c|}{$\begin{array}{l}\text { Magnetic forces alone present } \\
\left(M_{1} \rightarrow \infty\right)\end{array}$} \\
\hline & & $\left(R_{c}^{s}\right)_{\min }$ & $D a_{m}^{-1}$ & $\left(R_{c}^{s}\right)_{\min }$ & $D a_{m}^{-1}$ & $\left(R_{m c}\right)_{\min }$ & $D a_{m}^{-1}$ \\
\hline \multirow[t]{5}{*}{100} & 1 & 85346.407 & 453.989 & 156564.00 & 584.322 & 182235.193 & 361.681 \\
\hline & 1.5 & 85346.407 & 418.245 & 156564.00 & 559.73 & 182235.193 & 314.306 \\
\hline & 2 & 85346.407 & 382.502 & 156564.00 & 535.138 & 182235.193 & 266.931 \\
\hline & 2.5 & 85346.407 & 346.759 & 156564.00 & 510.546 & 182235.193 & 219.556 \\
\hline & 3 & 85346.407 & 311.015 & 156564.00 & 485.954 & 182235.193 & 172.180 \\
\hline \multirow[t]{5}{*}{0} & 1 & 42518.400 & 682.671 & 72526.50 & 786.888 & 97532.000 & 583.108 \\
\hline & 1.5 & 42518.400 & 663.668 & 72526.50 & 772.083 & 97532.000 & 558.433 \\
\hline & 2 & 42518.400 & 644.665 & 72526.50 & 757.279 & 97532.000 & 533.759 \\
\hline & 2.5 & 42518.400 & 625.663 & 72526.50 & 742.475 & 97532.000 & 509.085 \\
\hline & 3 & 42518.400 & 606.660 & 72526.50 & 727.670 & 97532.000 & 484.411 \\
\hline
\end{tabular}

$T a=10^{6}$ and $2 \times 10^{6}$ corroborate the above observations (see Tables $4 \mathrm{a}$ and $4 \mathrm{~b}$ ). As observed earlier, here a coupling between $D a^{-1}$ and $\Lambda_{m}$ is found such that $\left(R_{c}^{s}\right)_{\min }$ value remains unaltered for a fixed value of $\mathrm{Ta}$ and coincides with the one obtained previously by varying $\Lambda$ (see Tables $3 \mathrm{a}, 3 \mathrm{~b}$ ).

The variation of critical Darcy-Rayleigh number $R_{c}^{s}$ as a function of $\log _{10} H_{t}$ for different values of $\gamma$ with $T a=100, M_{1}=1, M_{3}=1, \Lambda=2$ and $D a^{-1}=$ 100 is presented in Fig. 4. We note that the curves of different $\gamma$ coalesce and asymptote to a single $R_{c}^{s}$ value when $H_{t}$ is small and the critical Rayleigh number remains almost independent of $H_{t}$ for $\gamma \geq 10$. This is because, for very small values of $H_{t}$ and higher values of $\gamma$ there is no significant transfer of heat between the fluid and solid phases, and hence the condition for the onset of convection is not affected by the properties of the solid phase. This corresponds to classical LTE limit. However, $R_{c}^{s}$ varies with $\gamma$ as the value of $H_{t}$ goes on increasing and remains independent of $H_{t}$ at higher values of $H_{t}$. The figure also indicates that for moderate and large values of $H_{t}$, the critical Rayleigh 
Table 4a Values of $\left(R_{c}^{s}\right)_{\min },\left(R_{m c}\right)_{\min }$ and $\Lambda_{m}$ for various vales of $D a^{-1}$ and $M_{1}$ when $M_{3}=1, \gamma=0.5$ and $T a=10^{6}$

\begin{tabular}{|c|c|c|c|c|c|c|c|}
\hline \multirow[t]{2}{*}{$H_{t}$} & \multirow[t]{2}{*}{$D a^{-1}$} & \multicolumn{2}{|c|}{$\begin{array}{l}\text { Simultaneous presence of } \\
\text { buoyancy and magnetic } \\
\text { forces }\left(M_{1}=1\right)\end{array}$} & \multicolumn{2}{|c|}{$\begin{array}{l}\text { Buoyancy forces alone present } \\
\left(M_{1}=0\right)\end{array}$} & \multicolumn{2}{|c|}{$\begin{array}{l}\text { Magnetic forces alone } \\
\text { present }\left(M_{1} \rightarrow \infty\right)\end{array}$} \\
\hline & & $\left(R_{c}^{s}\right)_{\min }$ & $\Lambda_{m}$ & $\left(R_{c}^{s}\right)_{\min }$ & $\Lambda_{m}$ & $\left(R_{m c}\right)_{\min }$ & $\Lambda_{m}$ \\
\hline \multirow[t]{5}{*}{100} & 10 & 60349.023 & 5.058 & 110707.454 & 8.904 & 128860.00 & 3.301 \\
\hline & 20 & 60349.023 & 4.918 & 110707.454 & 8.701 & 128860.00 & 3.195 \\
\hline & 30 & 60349.023 & 4.778 & 110707.454 & 8.498 & 128860.00 & 3.099 \\
\hline & 50 & 60349.023 & 4.498 & 110707.454 & 8.091 & 128860.00 & 2.879 \\
\hline & 100 & 60349.023 & 3.799 & 110707.454 & 7.075 & 128860.00 & 2.351 \\
\hline \multirow[t]{5}{*}{0} & 10 & 30065.000 & 13.145 & 51284.000 & 19.162 & 68966.00 & 8.860 \\
\hline & 20 & 30065.000 & 12.882 & 51284.000 & 18.824 & 68966.00 & 8.657 \\
\hline & 30 & 30065.000 & 12.619 & 51284.000 & 18.486 & 68966.00 & 8.455 \\
\hline & 50 & 30065.000 & 12.093 & 51284.000 & 17.811 & 68966.00 & 8.049 \\
\hline & 100 & 30065.000 & 10.777 & 51284.000 & 16.122 & 68966.00 & 7.036 \\
\hline
\end{tabular}

Table 4b Values of $\left(R_{c}^{s}\right)_{\min },\left(R_{m c}\right)_{\min }$ and $\Lambda_{m}$ for various vales of $D a^{-1}$ and $M_{1}$ when $M_{3}=1, \gamma=0.5$ and $T a=2 \times 10^{6}$

\begin{tabular}{|c|c|c|c|c|c|c|c|}
\hline \multirow[t]{2}{*}{$H_{t}$} & \multirow[t]{2}{*}{$D a^{-1}$} & \multicolumn{2}{|c|}{$\begin{array}{l}\text { Simultaneous presence of } \\
\text { buoyancy and magnetic forces } \\
\left(M_{1}=1\right)\end{array}$} & \multicolumn{2}{|c|}{$\begin{array}{l}\text { Buoyancy forces alone present } \\
\left(M_{1}=0\right)\end{array}$} & \multicolumn{2}{|c|}{$\begin{array}{l}\text { Magnetic forces alone } \\
\text { present }\left(M_{1} \rightarrow \infty\right)\end{array}$} \\
\hline & & $\left(R_{c}^{s}\right)_{\min }$ & $\Lambda_{m}$ & $\left(R_{c}^{s}\right)_{\min }$ & $\Lambda_{m}$ & $\left(R_{m c}\right)_{\min }$ & $\Lambda_{m}$ \\
\hline \multirow[t]{5}{*}{100} & 10 & 85346.407 & 7.211 & 156564.00 & 12.677 & 182235.193 & 4.712 \\
\hline & 20 & 85346.407 & 7.071 & 156564.00 & 12.474 & 182235.193 & 4.606 \\
\hline & 30 & 85346.407 & 6.931 & 156564.00 & 12.270 & 182235.193 & 4.501 \\
\hline & 50 & 85346.407 & 6.651 & 156564.00 & 11.814 & 182235.193 & 4.290 \\
\hline & 100 & 85346.407 & 5.952 & 156564.00 & 10.847 & 182235.193 & 3.762 \\
\hline \multirow[t]{5}{*}{0} & 10 & 42518.400 & 18.699 & 72526.50 & 27.238 & 97532.000 & 12.614 \\
\hline & 20 & 42518.400 & 18.436 & 72526.50 & 26.901 & 97532.000 & 12.411 \\
\hline & 30 & 42518.400 & 18.173 & 72526.50 & 26.563 & 97532.000 & 12.208 \\
\hline & 50 & 42518.400 & 17.647 & 72526.50 & 25.887 & 97532.000 & 11.803 \\
\hline & 100 & 42518.400 & 16.331 & 72526.50 & 24.199 & 97532.000 & 10.790 \\
\hline
\end{tabular}

number decreases with the increasing values of $\gamma$. In other words, increasing $\gamma$ is to hasten the onset of ferromagnetic convection because heat is transported to the system through both by solid and fluid phases. Figures 5 and 6 respectively show that increasing $M_{1}$ (i.e., increasing magnetic force) and decreasing $T a$ is to hasten the onset of ferromagnetic convection but for small values of $H_{t}$ the curves of different $M_{1}$ and $T a$ do not coalesce. A similar trend is noticed on the stability characteristics of the system with increasing $M_{3}$.
The variation of critical modified Rayleigh number $R_{M p c}^{s}$, which is defined in terms of the mean properties of the porous medium, is represented in Fig. 7 as a function of $\log _{10} H_{t}$ for different values of $\gamma$ for two values of $T a(=0,1000)$ with $M_{1}=1, M_{3}=1$, $D a^{-1}=100$ and $\Lambda=2$. From the figure, it is noted that $R_{M p c}^{s}$ approaches a common limit for different values of $\gamma$ as $H_{t} \rightarrow \infty$, however the approach to the common limit is different for different values of $T a$. It is also interesting to note that the critical Rayleigh number based on the mean properties remains almost 


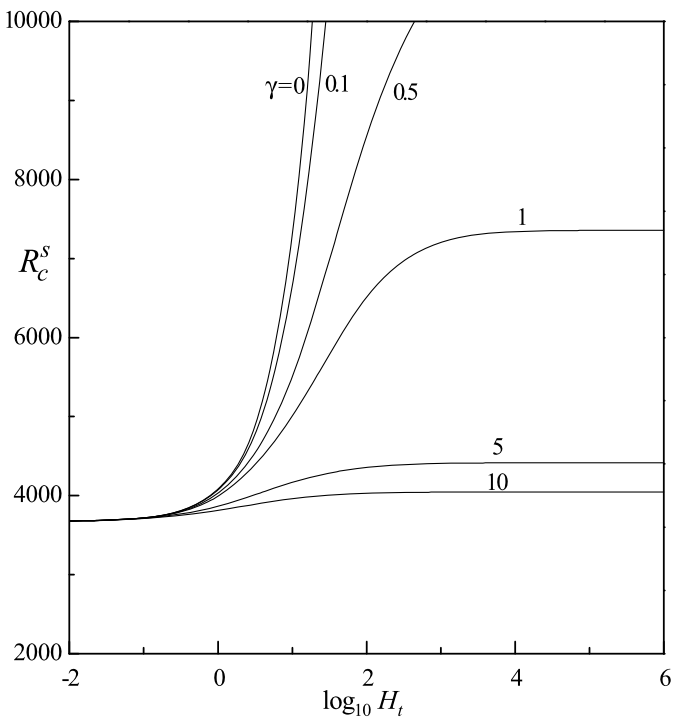

Fig. 4 Variation of $R_{c}^{s}$ with $\log _{10} H_{t}$ for different values of $\gamma$ with $T a=100, M_{1}=1, M_{3}=1, \Lambda=2$ and $D a^{-1}=100$

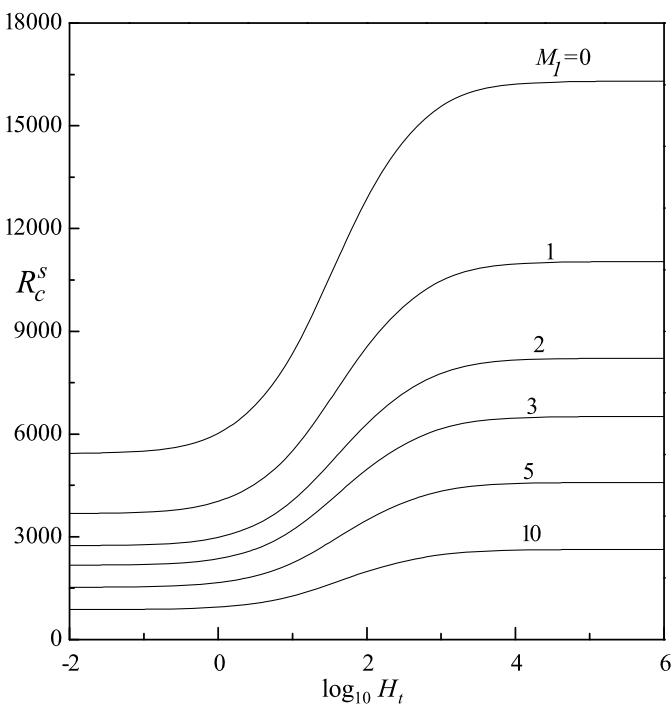

Fig. 5 Variation of $R_{c}^{s}$ with $\log _{10} H_{t}$ for different values of $M_{1}$ with $\gamma=0.5, T a=100, M_{3}=1, \Lambda=2$ and $D a^{-1}=100$

invariant for very small values of $H_{t}$ as well as large $\gamma$ indicating the complete suppression of ferromagnetic convection under these conditions.

It will be interesting and intuitive to know the response of the system when the magnetic forces alone are present (i.e., in the absence of buoyancy forces). In such a case, the magnetic Rayleigh number $R_{m}$ ( $\left.=R^{s} M_{1}\right)$ turns out to be the eigenvalue. From (27), we note that $\partial R_{m} / \partial T a>0$ and $\partial R_{m} / \partial M_{3}<0$. Thus

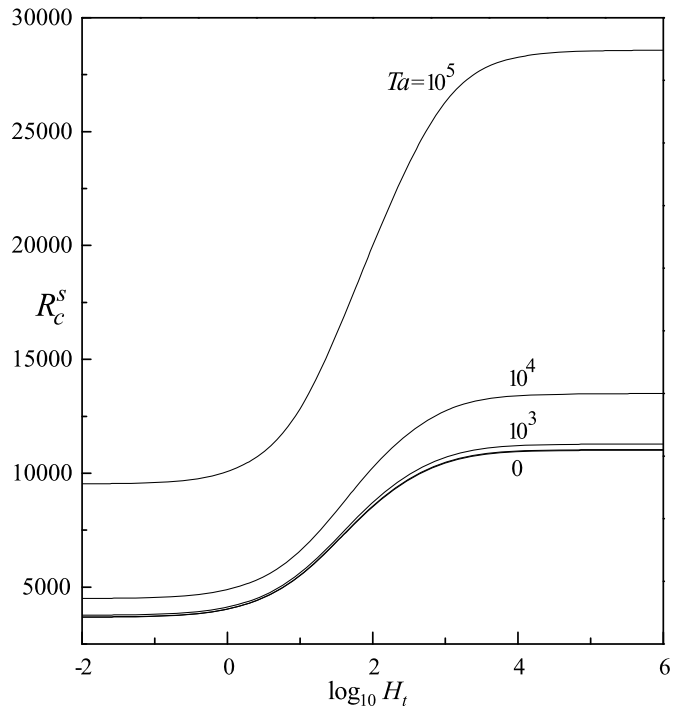

Fig. 6 Variation of $R_{c}^{s}$ with $\log _{10} H_{t}$ for different values of $T a$ with $\gamma=0.5, M_{1}=1, M_{3}=1, \Lambda=2$ and $D a^{-1}=100$

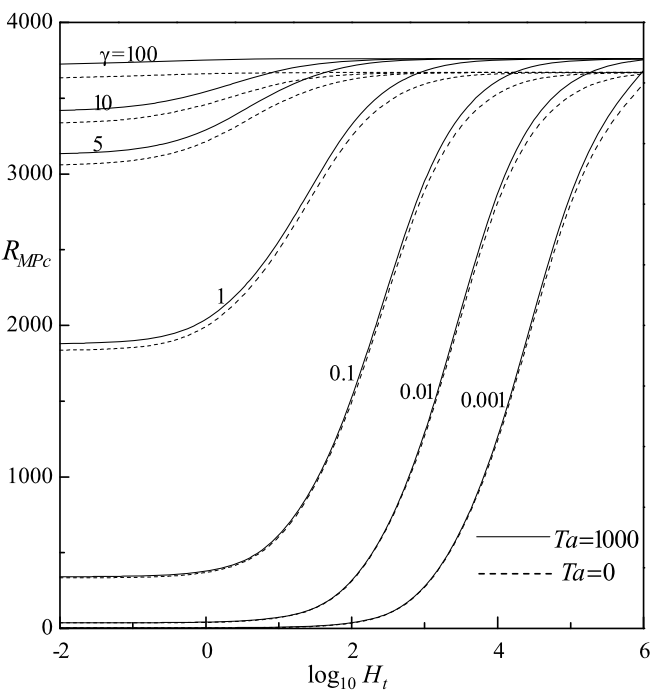

Fig. 7 Variation of $R_{M P c}^{s}$ with $\log _{10} H_{t}$ for different values of $\gamma$ and $T a$ with $M_{1}=1, M_{3}=1, \Lambda=2$ and $D a^{-1}=100$

increasing $T a$ is to make the system more stable, while increasing $M_{3}$ is to make the system more unstable. We further note that,

$$
\begin{aligned}
\frac{\partial R_{m}}{\partial D a^{-1}}= & \frac{\left(a^{2} M_{3}+\pi^{2}\right)\left\{H_{t}(1+\gamma)+\delta^{2}\right\} \delta^{2}}{a^{4} M_{3}\left(H_{t} \gamma+\delta^{2}\right)} \\
& \times\left[2 \delta^{2}-\frac{\pi^{2} T a+\delta^{2}\left(D a^{-1}+\delta^{2} \Lambda\right)^{2}}{\left(D a^{-1}+\delta^{2} \Lambda\right)^{2}}\right]
\end{aligned}
$$




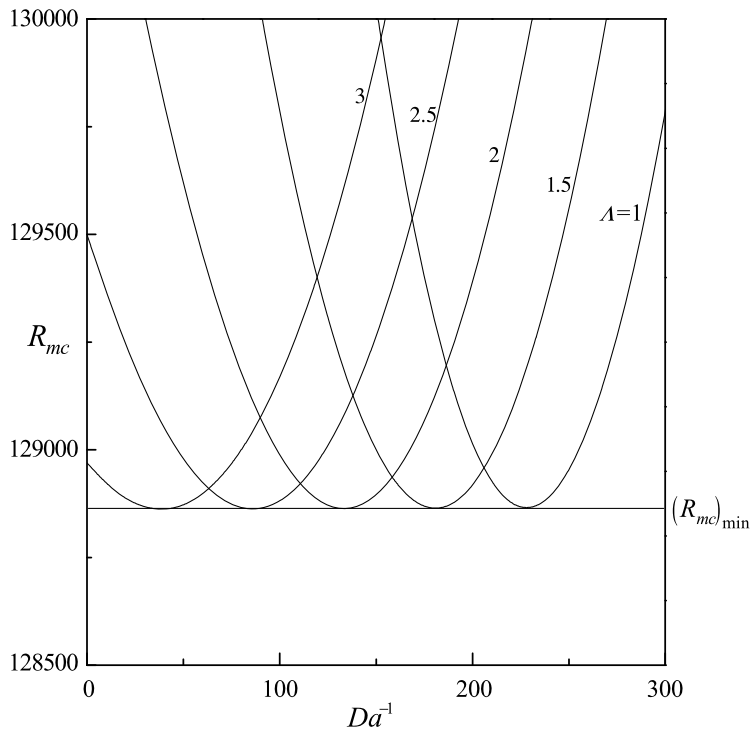

Fig. 8 Variation of $R_{m c}$ with $D a^{-1}$ for different values of $\Lambda$ with $\gamma=0.5, T a=10^{6}, M_{3}=1$ and $H_{t}=100$

$$
\begin{aligned}
\frac{\partial R_{m}}{\partial \Lambda}= & \frac{\left(a^{2} M_{3}+\pi^{2}\right)\left\{H_{t}(1+\gamma)+\delta^{2}\right\} \delta^{4}}{a^{4} M_{3}\left(H_{t} \gamma+\delta^{2}\right)} \\
& \times\left[2 \delta^{2}-\frac{\pi^{2} T a+\delta^{2}\left(D a^{-1}+\delta^{2} \Lambda\right)^{2}}{\left(D a^{-1}+\delta^{2} \Lambda\right)^{2}}\right] .
\end{aligned}
$$

It is seen that the right hand side of (50) and (51) may be either positive or negative depending on the choices of parametric values. In other words, $D a^{-1}$ and $\Lambda$ can have a destabilizing effect on the system even in this case. However, $R_{m}$ is an increasing function of $D a^{-1}$ and $\Lambda$ when $T a=0$ and thus their effect is to delay the onset of ferromagnetic convection in a ferromagnetic fluid saturated porous layer.

The variation of $R_{m c}$ (i.e., the critical value of $R_{m}$ with respect to the wave number $a$ ) as a function of $D a^{-1}$ for various values of $\Lambda$ and as a function of $\Lambda$ for various values of $D a^{-1}$ when $T a=10^{6}, M_{3}=1$, $\gamma=0.5$ and $H_{t}=100$ is shown in Figs. 8 and 9, respectively. From the figures, it is observed that $R_{m c}$ passes through a minimum with increasing $D a^{-1}$ and $\Lambda$ indicating their destabilizing effect on ferromagnetic convection in a rotating layer of ferromagnetic fluid saturated porous medium. Moreover, there is a coupling between the values of $D a^{-1}$ and $\Lambda$ in destabilizing the system with respect to these parameters. Also, the coupling between $\Lambda$ and $D a_{m}^{-1}$ or $D a^{-1}$ and $\Lambda_{m}$ is such that the $\left(R_{m c}\right)_{\min }$, the minimum value of $R_{m c}$ with respect to $D a^{-1}$ or $\Lambda$, as the case may be, is

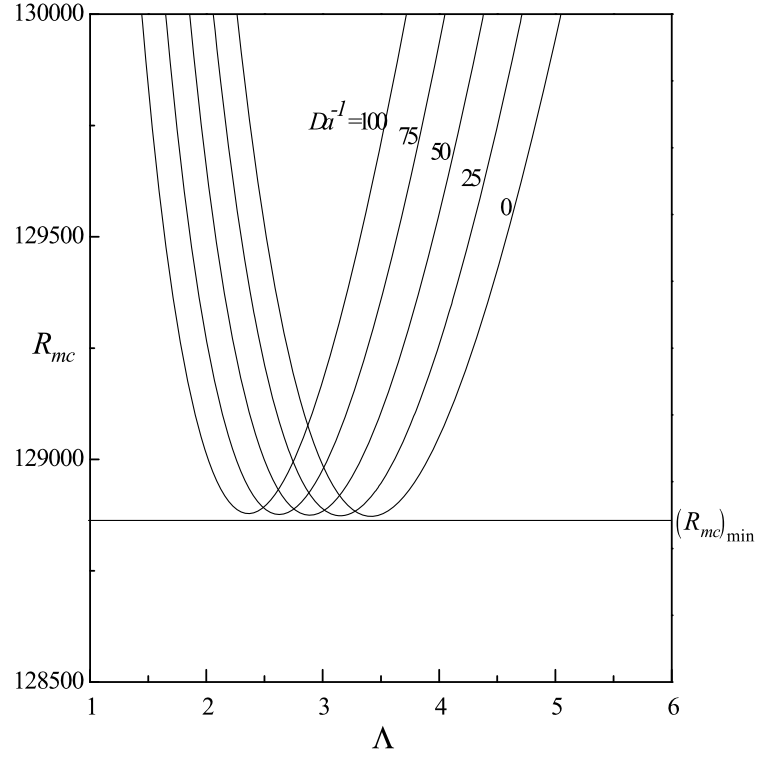

Fig. 9 Variation of $R_{m c}$ with $\Lambda$ for different values of $D a^{-1}$ with $T a=10^{6}, M_{3}=1, \gamma=0.5$ and $H_{t}=100$

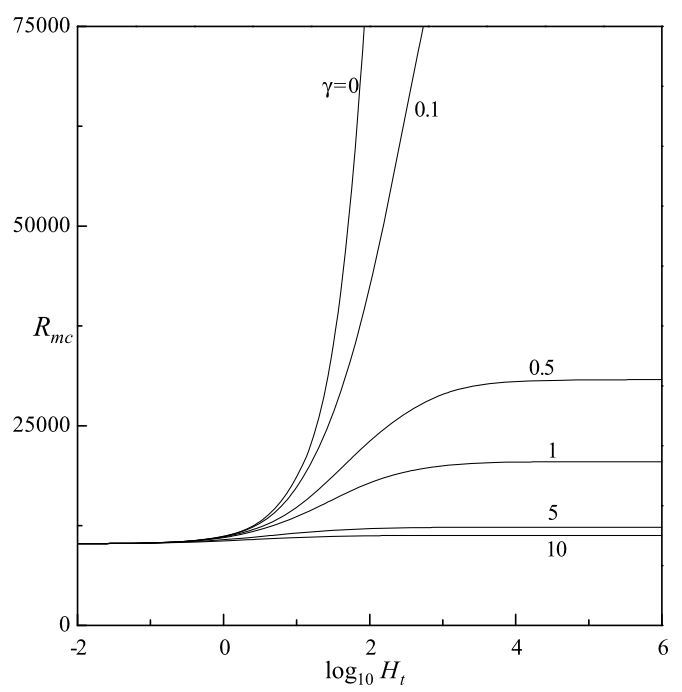

Fig. 10 Variation of $R_{m c}$ with $\log _{10} H_{t}$ for different values of $\gamma$ with $T a=100, M_{3}=1, \Lambda=2$ and $D a^{-1}=100$

the same for a fixed value of Taylor number. The values tabulated in Tables $3 \mathrm{a}, 3 \mathrm{~b}$ and $4 \mathrm{a}, 4 \mathrm{~b}$ for different values of $\Lambda$ and $D a^{-1}$ for two values of $T a=10^{6}$ and $2 \times 10^{6}$ confirm this result. Except for a quantitative change, the effect of $\gamma$ and $T a$ on $R_{m c}$ is akin to their behavior on $R_{c}^{s}$ (see Figs. 10 and 11).

To identify the similarities and differences between the magnetic mechanism alone and the combined ef- 


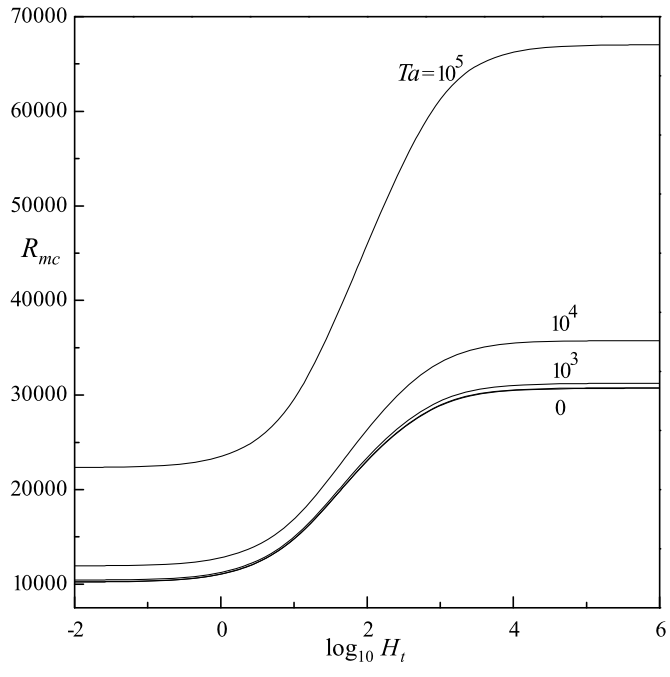

Fig. 11 Variation of $R_{m c}$ with $\log _{10} H_{t}$ for different values of Ta with $\gamma=0.5, M_{3}=1, \Lambda=2$ and $D a^{-1}=100$

fect of magnetic and buoyancy mechanisms on the onset of ferromagnetic convection in a rotating layer of porous medium, the values of $R_{m c}$ as well as $R_{c}^{s}$ and the corresponding critical wave numbers obtained as a function of $\log _{10} H_{t}$ are compared in Figs. 12 and 13, respectively for two values of $\gamma$ and $M_{3}$ when $T a=100, M_{1}=1, \Lambda=2$ and $D a^{-1}=100$. It is seen that $R_{m c}>R_{c}^{s}$ (see Fig. 12) and hence the system is more stabilizing when the magnetic mechanism alone is present (i.e., in the absence of buoyancy force). Similar is the case with the critical wave number (see Fig. 13). From the figures it is also observed that the effect of $\gamma$ and $M_{3}$ on $R_{m c}$ and $a_{c}$ is found to be more pronounced when the magnetic mechanism alone is present.

The values tabulated in Tables $3 \mathrm{a}, 3 \mathrm{~b}$ and $4 \mathrm{a}, 4 \mathrm{~b}$ for different values of $\Lambda$ and $D a^{-1}$ for two values of $T a$ $\left(=10^{6}\right.$ and $\left.2 \times 10^{6}\right)$ and $H_{t}(=100$ and 0$)$ also confirm the above observed behavior. From Tables $3 a, 3 b$ and $4 a, 4 b$, it is further seen that the results obtained when the buoyancy and magnetic forces are acting together or in isolation as well as for LTNE $\left(H_{t}=100\right)$ and LTE cases $\left(H_{t}=0\right)$ exhibit similar behavior. However, the values of $D a_{m}^{-1}$ for different values of $\Lambda$ and the values of $\Lambda_{m}$ for different values of $D a^{-1}$ as well as for LTNE and LTE cases for a fixed value of Taylor number are not the same when these forces are acting together and in isolation despite the expressions for $D a_{m}^{-1}$ and $\Lambda_{m}$ (cf. (48) and (49)) are the same in all the cases. This is because of change in the values of

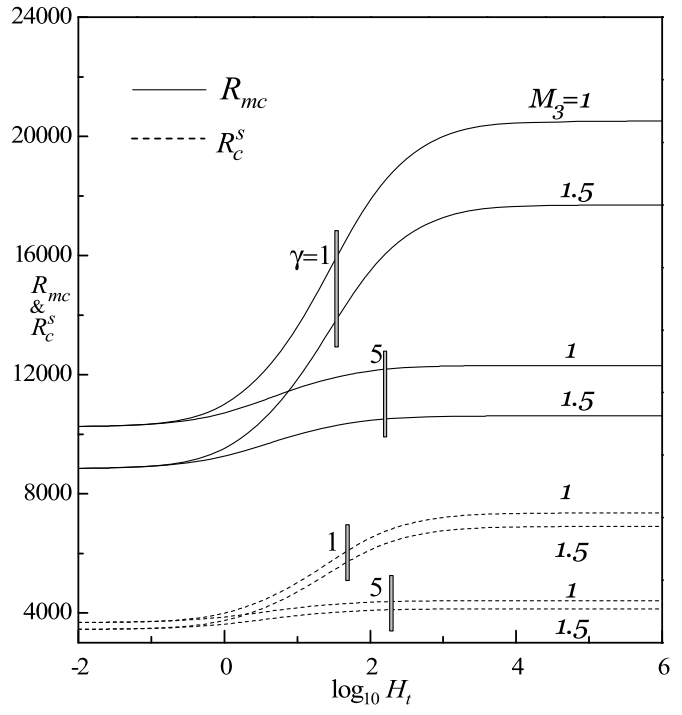

Fig. 12 Variation of $R_{c}^{s}$ and $R_{m c}$ with $\log _{10} H_{t}$ for two values of $\gamma$ and $M_{3}$ with $T a=100, M_{1}=1, \Lambda=2$ and $D a^{-1}=100$

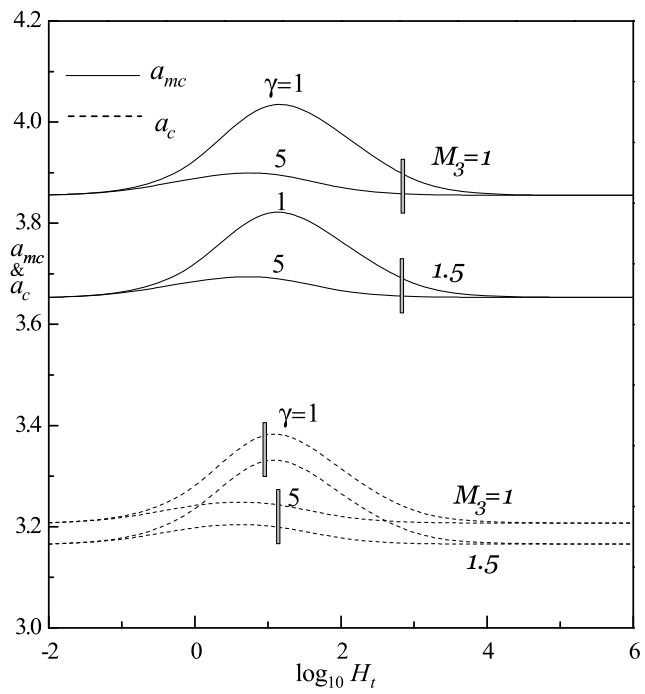

Fig. 13 Variation of $a_{m c}$ and $a_{c}$ with $\log _{10} H_{t}$ for different values of $\gamma$ and $M_{3}$ with $T a=100, M_{1}=1, \Lambda=2$ and $D a^{-1}=100$

critical wave number in all these different cases considered. Also, we note that

$$
\begin{aligned}
{\left[\left(R_{m c}\right)_{\min }\right]_{\text {magnetic }} } & >\left[\left(R_{c}^{s}\right)_{\min }\right]_{\text {buoyancy }} \\
& >\left[\left(R_{c}^{s}\right)_{\min }\right]_{\text {buoyancy+magnetic }}
\end{aligned}
$$

for a fixed value of Taylor number in both LTNE and LTE cases. This indicates that the onset of ferromagnetic convection in a rotating porous layer is delayed 
the most when the magnetic forces alone are present. In other words, the combined effect of buoyancy and magnetic forces is to reinforce together and to hasten the onset of ferromagnetic convection in a rotating ferrofluid saturated porous layer. Also, values of $\left(R_{c}^{s}\right)_{\text {min }}$ for the LTNE case are higher than those of the LTE case. In addition,

$$
\begin{aligned}
{\left[\mathrm{Da}_{m}^{-1}\right]_{\text {buoyancy }} } & >\left[D a_{m}^{-1}\right]_{\text {buoyancy+magnetic }} \\
& >\left[D a_{m}^{-1}\right]_{\text {magnetic }}
\end{aligned}
$$

for all values of $\Lambda$ considered, and

$$
\begin{aligned}
{\left[\Lambda_{m}\right]_{\text {buoyancy }} } & >\left[\Lambda_{m}\right]_{\text {buoyancy }}+\text { magnetic } \\
& >\left[\Lambda_{m}\right]_{\text {magnetic }}
\end{aligned}
$$

for all values of $D a^{-1}$ considered. It is also seen that $D a_{m}^{-1}$ and $\Lambda_{m}$ values for the LTNE case are lower than those of LTE case.

The critical wave number remains the same for different values of $\gamma$ in both the small and large $H_{t}$ limits and this is evident from Fig. 13. However, at moderate values of $H_{t}$, the critical wave number reaches its peak value and increasing $\gamma$ decreases the value of $a_{c}$. In other words, increase in the value of $\gamma$ is to enlarge the size of convection cells only at moderate values of $H_{t}$ and the size of convection cells remains independent of $\gamma$ when $H_{t} \ll 1$ and $H_{t} \gg 1$. We note that increasing $M_{3}$ is to decrease the critical wave number (i.e., to diminish the size of convection cells). Further inspection of these figures reveals that the value of critical wave number is higher when the onset of ferromagnetic convection is due to magnetic forces alone as compared to the simultaneous presence of buoyancy and magnetic forces.

\section{Conclusions}

The criterion for the onset of thermal convection in a ferromagnetic fluid saturated horizontal rotating Brinkman porous layer in the presence of a uniform magnetic field with a thermal non-equilibrium model is studied. Condition for the onset of stationary and oscillatory onset is obtained analytically. Contrary to their usual influence on the onset of convection in the absence of rotation, both $D a^{-1}$ and $\Lambda$ show destabilizing effects on the stationary onset at high rotation rates. In other words, decrease in the permeability of the porous medium and increase in the viscosity of the fluid have destabilizing effect on the system. The numerically computed double minimum of $R^{s}$ or $R_{m}$ with respect to $a$ and $D a^{-1}$ or $a$ and $\Lambda$, denoted by $\left(R_{c}^{s}\right)_{\min }$ or $\left(R_{m c}\right)_{\min }$, as the case may be, shows that there is a coupling between the values of $D a_{m}^{-1}$ and $\Lambda$ or $\Lambda_{m}$ and $D a^{-1}$ such that $\left(R_{c}^{s}\right)_{\text {min }}$ and $\left(R_{m c}\right)_{\text {min }}$ remain the same for a fixed value of Taylor number. Increase in the value of Taylor number $T a$ is to reinforce stability on the system and the LTNE case is found to have more stabilizing effect on the system as compared to the LTE case. The porosity modified conductivity ratio $\gamma$ has no effect on the onset of ferromagnetic convection in the small- $H_{t}$ limit, while for the other values of $H_{t}$ increase in the value of $\gamma$ is to hasten the onset of ferromagnetic convection. At higher values of $\gamma$, the onset of convection remains independent of $H_{t}$ and tends to classical LTE case. The effect of increase in the magnetic number $M_{1}$ and the nonlinearity of magnetization parameter $M_{3}$ is to advance the onset of ferromagnetic convection. The system is more stabilizing when the magnetic force alone is present and in that case $M_{3}$ and $\gamma$ show prominence effect on the onset of ferromagnetic convection when compared to the simultaneous presence of buoyancy and magnetic forces. The critical Rayleigh number $R_{M p c}^{s}$ based on the mean properties of the porous medium approaches a common limit as $H_{t} \rightarrow \infty$ for different values of $\gamma$ and for any fixed value of $D a^{-1}$, $\Lambda, T a, M_{1}$ and $M_{3}$. The critical wave number for different values of $\gamma$ in the small- $H_{t}$ and large- $H_{t}$ limits coincide, but attain a maximum value at the intermediate values of $H_{t}$ and in that case increasing $\gamma$ is to decrease the critical wave number. The critical wave number is higher when the onset of convection is only due to magnetic forces as compared to the simultaneous presence of buoyancy and magnetic forces.

Acknowledgements The authors (R.G.) and (M.R.) wish to thank the Principals of their respective colleges for their encouragement. We thank the reviewers for their encouraging comments.

\section{Appendix}

The expressions for $\Delta_{1}$ and $\Delta_{2}$ appearing in (21) are:

$$
\begin{aligned}
\Delta_{1}= & \left(\delta^{4}+\alpha^{2} \omega^{2}\right)\left\{\pi^{2} \operatorname{Pr}^{2} \operatorname{Ta}\left(\operatorname{Pr} \delta^{4} \Lambda+\omega^{2}\right)\right. \\
& +\delta^{2}\left(\operatorname{Pr}^{3} \delta^{8} \Lambda^{3}+\operatorname{Pr} \delta^{4} \Lambda \omega^{2}\right.
\end{aligned}
$$




$$
\begin{aligned}
& \left.\left.-P r^{2} \delta^{4} \Lambda^{2} \omega^{2}-\omega^{4}\right)\right\} \\
& +\left(D a^{-1}\right)^{3} \operatorname{Pr}^{3} \delta^{4} H_{t}^{2} \gamma(1+\gamma)+\left(D a^{-1}\right)^{3} \\
& \times \operatorname{Pr}^{3} \delta^{2}\left\{\delta^{6}+\alpha^{2} \delta^{2} \omega^{2}\right. \\
& \left.+H_{t}(1+2 \gamma) \delta^{4}+\alpha^{2} \omega^{2}\right\} \\
& +H_{t}^{2} \gamma \delta^{2}\left(\operatorname{Pr}^{2} \delta^{4} \Lambda+\omega^{2}\right) \\
& \times\left\{\operatorname{Pr}(1+\gamma) \delta^{4} \Lambda-(\alpha+\gamma) \omega^{2}\right\} \\
& +H_{t}^{2} \gamma \pi^{2} \operatorname{Pr}^{2} \operatorname{Ta}\left\{\operatorname{Pr}(1+\gamma) \delta^{4} \Lambda+(\alpha+\gamma) \omega^{2}\right\} \\
& +H_{t} \delta^{2} \pi^{2} \operatorname{Pr}^{2} \operatorname{Ta}\left\{\delta^{4} \Lambda \operatorname{Pr}(1+2 \gamma)\right. \\
& \left.+\left(2 \gamma+\operatorname{Pr}^{2} \Lambda\right) \omega^{2}\right\} \\
& +H_{t} \delta^{4}\left(\operatorname{Pr}^{2} \delta^{4} \Lambda^{2}+\omega^{2}\right) \\
& \left\{\operatorname{Pr} \Lambda\left(\delta^{4}+2 \gamma \delta^{4}+\alpha^{2} \omega^{2}\right)-2 \gamma \omega^{2}\right\} \\
& +\left(D a^{-1}\right)^{2} \operatorname{Pr}^{2} \delta^{2}\left(3 \operatorname{Pr} \delta^{4} \Lambda-\omega^{2}\right)\left(\delta^{4}+\alpha^{2} \omega^{2}\right) \\
& +\left(D a^{-1}\right)^{2} \delta^{2} H_{t}^{2} \gamma\left\{3 \operatorname{Pr} \delta^{4} \Lambda(1+\gamma)\right. \\
& \left.-(\alpha+\gamma) \omega^{2}\right\} \\
& +\left(D a^{-1}\right)^{2} \delta^{4} H_{t}\left\{3 \operatorname{Pr} \Lambda\left(\delta^{4}+2 \gamma \delta^{4}+\alpha^{2} \omega^{2}\right)\right. \\
& \left.-2 \gamma \omega^{2}\right\} \\
& +D a^{-1} \operatorname{Pr} \delta^{2}\left(\delta^{4}+\alpha^{2} \omega^{2}\right)\left\{\pi^{2} \operatorname{Pr}^{2} T a\right. \\
& \left.+\delta^{2}\left(3 \operatorname{Pr}^{2} \delta^{4} \Lambda^{2}+\omega^{2}-2 \operatorname{Pr} \Lambda \omega^{2}\right)\right\} \\
& +D a^{-1} \delta^{2} H_{t}^{2} \gamma \pi^{2} \operatorname{Pr}^{2} \operatorname{Ta}(1+\gamma) \\
& +D a^{-1} \delta^{4} H_{t}^{2} \gamma\left\{(1+\gamma)\left(3 \operatorname{Pr}^{2} \delta^{4} \Lambda^{2}+\omega^{2}\right)\right. \\
& \left.-2 \operatorname{Pr}(\alpha+\gamma) \Lambda \omega^{2}\right\} \\
& +D a^{-1} H_{t} \pi^{2} \operatorname{Pr}^{2} \operatorname{Ta}\left\{(1+2 \gamma) \delta^{4}+\alpha^{2} \omega^{2}\right\} \\
& +D a^{-1} H_{t} \delta^{2}\left\{\left(\delta^{4}+2 \gamma \delta^{4}+\alpha^{2} \omega^{2}\right)\right. \\
& \left.\times\left(3 \operatorname{Pr}^{2} \delta^{4} \Lambda^{2}+\omega^{2}\right) P r-4 \gamma \delta^{4} \Lambda \operatorname{Pr}^{2} \omega^{2}\right\} \\
& \Delta_{2}=\left(D a^{-1}\right)^{3} \operatorname{Pr}^{3} \delta^{2}\left\{H_{t}^{2} \gamma(\alpha+\gamma)+2 H_{t} \gamma \delta^{2}+\delta^{4}\right. \\
& \left.+\alpha^{2} \omega^{2}\right\}+\delta^{2}\left(\delta^{4}+\alpha^{2} \omega^{2}\right)\left\{\pi^{2} \operatorname{Pr}^{2} T a\right. \\
& \times(\operatorname{Pr} \Lambda-1)\} \\
& +\delta^{4}\left(\delta^{4}+\alpha^{2} \omega^{2}\right)(1+\operatorname{Pr} \Lambda)\left(\delta^{4} \operatorname{Pr}^{2} \Lambda^{2}+\omega^{2}\right) \\
& +\delta^{2} H_{t}^{2} \gamma\left\{\pi^{2} \operatorname{Pr}^{2} \operatorname{Ta}(\gamma(\operatorname{Pr} \Lambda-1)-1\right. \\
& +\alpha \Lambda P r)\} \\
& +\delta^{4} H_{t}^{2} \gamma(1+\gamma+\operatorname{Pr} \alpha \Lambda+\operatorname{Pr} \gamma \Lambda)
\end{aligned}
$$

$$
\begin{aligned}
& \left(\operatorname{Pr}^{2} \delta^{4} \Lambda^{2}+\omega^{2}\right)+H_{t}\left\{\pi^{2} \operatorname{Pr}^{2} \operatorname{Ta}(2 \gamma(\Lambda P r-1)\right. \\
& \text {-1) } \left.\delta^{4}-\alpha^{2} \omega^{2}\right\} \\
& +H_{t} \delta^{2}\left\{(1+2 \gamma+2 \gamma \operatorname{Pr} \Lambda) \delta^{4}+\alpha^{2} \omega^{2}\right\} \\
& \times\left(\operatorname{Pr}^{2} \delta^{4} \Lambda^{2}+\omega^{2}\right) \\
& +\left(D a^{-1}\right)^{2} \operatorname{Pr}^{2} \delta^{4}\left\{H_{t}^{2} \gamma(1+\gamma+3 \operatorname{Pr} \alpha \Lambda\right. \\
& +3 \operatorname{Pr} \gamma \Lambda)\} \\
& +\left(D a^{-1}\right)^{2} \operatorname{Pr}^{2}\left\{\delta^{4}(1+3 \operatorname{Pr} \Lambda)\left(\delta^{4}+\alpha^{2} \omega^{2}\right)\right. \\
& \left.+H_{t}\left((1+2 \gamma+6 \operatorname{Pr} \gamma \Lambda) \delta^{6}\right)+\alpha^{2} \omega^{2} \delta^{2}\right\} \\
& +D a^{-1} \operatorname{Pr}\left(\delta^{4}+\alpha^{2} \omega^{2}\right)\left\{\pi^{2} \operatorname{Pr}^{2} T a\right. \\
& \left.+\operatorname{Pr} \delta^{6} \Lambda(2+3 \operatorname{Pr} \Lambda)+\omega^{2} \delta^{2}\right\} \\
& +2 D a^{-1} H_{t} \pi^{2} \operatorname{Pr}^{2} T a \delta^{2} \\
& +2 D a^{-1} H_{t} \delta^{4}\left\{\delta^{4}\left(\gamma \omega^{2}+3 \operatorname{Pr}^{2} \delta^{4} \Lambda\right)\right. \\
& \left.+\operatorname{Pr} \Lambda\left(\delta^{4}+2 \gamma \delta^{4}+\alpha^{2} \omega^{2}\right)\right\} \\
& +D a^{-1} H_{t}^{2} \gamma\left\{\pi^{2} \operatorname{Pr}^{2} \operatorname{Ta}(\alpha+\gamma)\right\} \\
& +D a^{-1} H_{t}^{2} \gamma \delta^{2}\left\{\delta^{4} \Lambda \operatorname{Pr}(2+2 \gamma\right. \\
& \left.+3 \operatorname{Pr} \alpha \Lambda+3 \operatorname{Pr} \gamma \Lambda)+(\alpha+\gamma) \omega^{2}\right\} \text {. }
\end{aligned}
$$

\section{References}

1. Rosensweig RE (1985) Ferrohydrodynamics. Cambridge University Press, Cambridge, London

2. Berkovsky BM, Medvedev VF, Krakov MS (1993) Magnetic fluids, engineering applications. Oxford University Press, New York

3. Blums E, Cebers A, Maiorov MM (1997) Magnetic fluids. de Gruyter, New York

4. Hergt R, Andrä W, Ambly CG, Hilger I, Kaiser WA, Richter U, Schmidt HG (1998) Physical limitations of hypothermia using magnetite fine particles. IEEE Trans Magn 34:3745-3754

5. Alexiou C, Arnold W, Hulin P, Klein R, Schmidt A, Bergemann C, Parak FG (2001) Therapeutic efficacy of ferrofluid bound anticancer agent. Magnetohydrodynamics 37:318-322

6. Ganguly R, Sen S, Puri IK (2004) Heat transfer augmentation using a magnetic fluid under the influence of a line dipole. J Magn Magn Mater 271:63-73

7. Odenbach S (2004) Recent progress in magnetic fluid research. J Phys Condens Matter 16:R1135-R1150

8. Rosensweig RE, Zahn M, Volger T (1978) Stabilization of fluid penetration through a porous medium using magnetizable fluids. In: Berkovsky B (ed) Thermomechanics of magnetic fluids. Hemisphere, Washington, pp 195-211 
9. Zhan M, Rosensweig RE (1980) Stability of magnetic fluid penetration through a porous medium with uniform magnetic field oblique to the interface. IEEE Trans Magn $16: 275-282$

10. Vaidyanathan G, Sekar R, Balasubramanian R (1991) Ferroconvective instability of fluids saturating a porous medium. Int J Eng Sci 29:1259-1267

11. Borglin SE, Mordis J, Oldenburg CM (2000) Experimental studies of the flow of ferrofluid in porous media. Transp Porous Media 41:61-80

12. Sunil Mahajan A (2009) Nonlinear stability analysis for thermoconvective magnetized ferrofluid saturating a porous medium. Transp Porous Media 76:327-343

13. Shivakumara IS, Nanjundappa CE, Ravisha M (2008) Thermomagnetic convection in a magnetic nanofluids fluid saturated porous medium. Int $\mathrm{J}$ Appl Math Eng Sci 2(2):157-170

14. Shivakumara IS, Nanjundappa CE, Ravisha M (2009) Effect of boundary conditions on the onset of thermomagnetic convection in a ferrofluid saturated porous medium. J Heat Transf 131:101003

15. Nanjundappa CE, Shivakumara IS, Ravisha M (2010) The onset of buoyancy-driven convection in a ferromagnetic fluid saturated porous medium. Meccanica 45:213-226

16. Rees DAS, Pop I (2005) Local thermal non-equilibrium in porous medium convection. In: Ingham DB, Pop I (eds) Transp phenomena in porous med, vol III. Elsevier, Oxford, pp 147-173

17. Nield DA, Bejan A (2006) Convection in porous media, 3rd edn. Springer, New York

18. Shivakumara IS, Mamatha AL, Ravisha M (2010) Boundary and thermal non-equilibrium effects on the onset of Darcy-Brinkman convection in a porous layer. J Eng Math 67:317-328

19. Sunil PS, Mahajan A (2010) Nonlinear ferroconvection in a porous layer using a thermal nonequilibrium model. Top Rev Porous Med Int J 1:105-121

20. Lee J, Shivakumara IS, Ravisha M (2011) Effect of thermal non-equilibrium on convective instability in a ferromagnetic fluid saturated porous medium. Transp Porous Media 86:103-124
21. Shivakumara IS, Lee J, Ravisha M, Gangadhara Reddy $\mathrm{R}$ (2011) The onset of Brinkman ferroconvection using a thermal non-equilibrium model. Int $\mathrm{J}$ Heat Mass Transf 54:2116-2125

22. Shivakumara IS, Lee J, Ravisha M, Gangadhara Reddy R (2012) The effects of local thermal nonequilibrium and MFD viscosity on the onset of Brinkman ferroconvection. Meccanica 47:1359-1378

23. Sekar R, Vaidyanathan G, Ramanathan A (1993) The ferroconvection in fluids saturating a rotating densely packed porous medium. Int J Eng Sci 31(2):241-250

24. Vaidyanathan G, Sekar R, Vasanthakumari R, Ramanathan A (2002) The effect of magnetic field dependent viscosity on ferroconvection in a rotating sparsely distributed porous medium. J Magn Magn Mater 250:65-76

25. Sunil PS, Mahajan A (2009) A nonlinear stability analysis for rotating magnetized ferrofluid heated from below saturating a porous medium. Z Angew Math Phys 60:344-362

26. Shivakumara IS, Lee J, Nanjundappa CE, Ravisha M (2011) Ferromagnetic convection in a rotating ferrofluid saturated porous layer. Transp Porous Media 87:251-273

27. Straughan B (2006) Global nonlinear stability in porous convection with a thermal non-equilibrium model. Proc $\mathrm{R}$ Soc A 462:409-418

28. Malashetty MS, Mahantesh S, Kulkarni S (2007) Thermal convection in a rotating porous layer using a thermal nonequilibrium model. Phys Fluids 19(5):054102

29. Sunil PS, Mahajan A (2011) Onset of Darcy-Brinkman ferroconvection in a rotating porous layer using a thermal nonequilibrium model: a nonlinear stability analysis. Transp Porous Media 88:421-439

30. Finlayson BA (1970) Convective instability of ferromagnetic fluids. J Fluid Mech 40:753-767

31. Shivakumara IS, Savitha MN, Krishna B, Chavaraddi DN (2009) Bifurcation analysis for thermal convection in a rotating porous layer. Meccanica 44:225-238

32. Palm E, Tyvand PA (1984) Thermal convection in a rotating porous layer. Z Angew Math Phys 35:122-133

33. Chandrasekhar S (1961) Hydrodynamic and hydromagnetic stability. Oxford University Press, London 